\title{
Large Eddy Simulations of Isothermal Confined Swirling Flow in an Industrial Gas-turbine
}

\author{
G. Bulat ${ }^{\mathrm{a}}$, W. P. Jones ${ }^{\mathrm{b}}$, S. Navarro-Martinez ${ }^{\mathrm{b}}$ \\ ${ }^{a}$ Siemens Industrial Turbomachinery Ltd., Waterside South, Lincoln LN5 7FD, UK \\ ${ }^{b}$ Department of Mechanical Engineering, Imperial College London, Exhibition Road, London \\ SW7 2AZ, UK
}

\begin{abstract}
The paper describes the results of a computational study of the strongly swirling isothermal flow in the combustion chamber of an industrial gas turbine. The flow field characteristics are computed using Large Eddy Simulation in conjunction with a dynamic version of the Smagorinsky model for the sub-grid-scale stresses. Grid refinement studies demonstrate that the results are essentially grid independent. The LES results are compared with an extensive set of measurements and the agreement with these is overall good. The method is shown to be capable of reproducing the observed Precessing Vortex and Central Vortex Cores and the profiles of mean and rms velocities are found to be captured to a good accuracy. The overall flow structure is shown to be virtually independent of Reynolds number. Keywords:

LES, Swirl-Flows, Iso-thermal, Coherent Structures, PVC
\end{abstract}

\section{Introduction}

Swirling flows are used in many industrial applications because of their tendency to enhance mixing and to induce regions of strong flow recirculation. In 
combustion chambers the result is compact flame and improved flame control characteristics, thus resulting in an improved combustion efficiency and low pollutant emissions.

The flow structure in confined strongly swirling flows is complex with large radial and axial pressure gradients arising. Typically the pressure in the central core is low but rises with increasing distance. This adverse pressure gradient results in vortex breakdown resulting in a reversal of the flow direction with associated high turbulence intensities and enhanced mixing. The flow structure is essentially determined by swirl number, the ratio of the axial flow rates of azimuthal momentum and axial momentum. Industrial combustion applications involving swirling flows usually operate at high swirl numbers to take full advantage of enhanced mixing. The main advantage of using a high swirl number is that the onset of the vortex breakdown region results in a shorter flame, high turbulence intensities (hence enhanced mixing between reactants and oxidant) and improved flame stability by aerodynamic anchoring of the flame.

Detailed reviews of vortex breakdown have been provided by a number of authors, including Hall (1972); Leibovich (1978); Escudier (1988); Lucca-Negro and O'Doherty (2001). Vortex breakdown is often accompanied by large scale unsteady structures, a Precessing Vortex Core (PVC), Syred (2006) and a Central Vortex Core (CVC). In the latter the flow recesses about the axis of symmetry. Secondary to the vortex breakdown the flow downstream of its occurrence is determined by the swirl number. At sufficiently high levels a columnar Rankine type vortex (CVC) can form with reverse flow arising along the entire axis of the device. Such a flow is termed sub-critical, Squire (1962). In Escudier et al. (2006) it was shown that the outlet geometry can have a significant influence over all the 
flow field. For sub-critical flow the downstream conditions are determined by the area ratio and may influence the vortex breakdown.

There have been many previous computational studies of swirling flows, with earlier work involving Reynolds averaged approaches (RANS) whilst more recent efforts have focussed on Large Eddy Simulation (LES). It is now well known RANS based eddy viscosity models, such as the $k-\epsilon$ model perform very poorly in strongly swirling flows. Second moment closures fare somewhat better, see for example Craft et al. (2008), although even these appear to be incapable of accurately reproducing the unsteady large scale structures that appear in strongly swirling confined flows. Both LES and unsteady RANS were applied in Dunham et al. (2009) to model the flow in a single swirl stream fuel injector where the capability of LES to reproduce flow characteristics was shown to be in excellent agreement with measurements. The use of URANS on the other hand led to identification of the vortical structures at the swirler exit but the predicted amplitudes and frequencies were not in accord with the measurements. In Jochmann et al. (2006) URANS with alternatively the $k-\epsilon$ model and a second moment was applied to the computation of axisymmetric vortex breakdown. The two models gave quite different results with the eddy viscosity model tending to damp the observed spiral shaped flow structures. Both models reproduced qualitatively the overall flow structure although the computed axial velocity profiles were in relatively poor agreement with measurements.

Several recent LES studies have been completed where comparisons with measured data have shown it capable of capturing different aspects of the flow. Extensive experimental data for both burning and non-burning conditions has been obtained in the Preccinsta experimental burner, Weigand et al. (2006a); Meier 
et al. (2007a), a Turbomeca radial burner operated at atmospheric conditions. Detailed LES for the reacting and isothermal case have been completed. These include LES using a compressible unstructured solver in conjunction with an acoustic analysis, Selle et al. (2004); Roux et al. (2005) where it was found that the PVC mode was very strong for the cold flow but disappeared with combustion taking place. The acoustic structure revealed by LES matches exactly the results of the acoustic solver for the $588 \mathrm{~Hz}$ mode. Excellent agreement was found between velocity fields arising from the LES and the LDV measurements.

In the present paper LES is applied to the isothermal strongly swirling confined flow within the Siemens SGT-100 combustion chamber at different Reynolds numbers, $\mathcal{O}\left(10^{5}\right)$. The results are compared with measurements arising from the TURCHEMI project Stopper et al. (2013). The present study includes several test cases to confirm the most suitable model parameters for complex wall-bounded flows within practical geometries. The influence of local mesh redistribution and mesh refinement is also investigated. Particular attention is given to the simulation of the coherent structures present within the combustor. The results of a LES study of the reacting case in the same geometry is described in Bulat et al. (2013, 2014).

\section{Experimental Set-up}

The SGT-100 is the smallest combustor from the Siemens range with a power up to $1 \mathrm{MW}$. Six combustors are used in the SGT-100 engine with a nominal power rating of up to 5.7MW for mechanical drive applications (twin-shaft) and a nominal power rating up to $5.4 \mathrm{MW}$ for power generation (single shaft) A slightly modified version of the SGT-100 Dry Low Emission combustor operating at 3 bar 
pressure conditions was selected as a test case. The SGT-100 combustor, shown in figure 1 was studied experimentally in the high pressure facility at the German Aerospace Centre (DLR) Stuttgart, Germany, Stopper et al. (2009), Stopper et al. (2010) and Stopper (2011a).

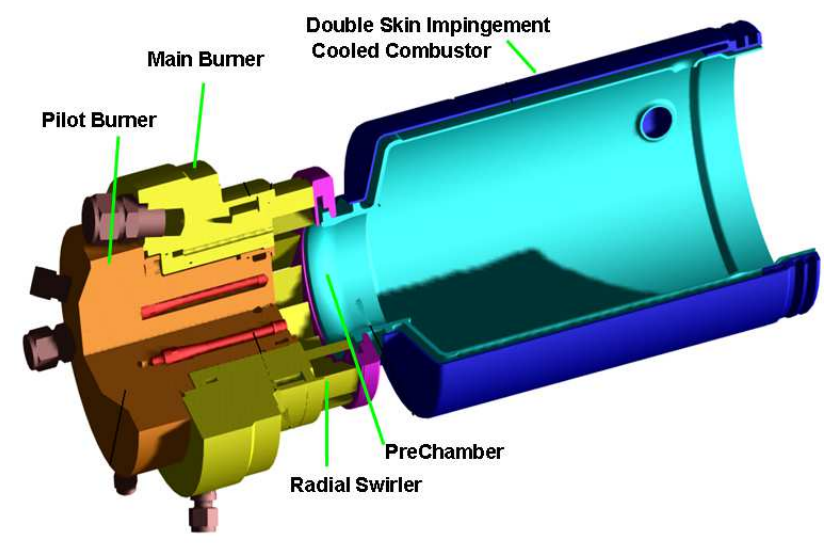

(a) Generic Siemens DLE combustor technology

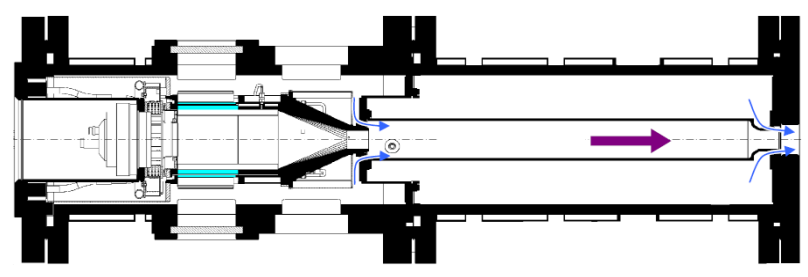

(b) Schematic of the Combustion Chamber rig

Figure 1: Experimental high pressure rig at DLR Stuttgart for the SGT-100 burner

The experimental setup comprises the burner, combustion chamber and a transition duct into an exhaust pipe. The square central section was required for laser access, so that the laser sheet is not distorted due to the cylindrical face of the original combustion can. The expansion ratio at the exit of the burner and the chamber is that of the original SGT-100 combustion can. The width of the side of the experimental chamber was equivalent to the diameter of the SGT-100 com- 
bustor and the length of the experimental chamber was retained as per the original cylindrical chamber. The length of the exhaust pipe was not related to the engine, but were determined by experimental constraints. The diameter of the exhaust pipe is $0.1 \mathrm{~m}$ and is twice the diameter of the combustor exit duct.

After passing through the swirler vanes, the flow turns through a right angle into the pre-chamber, followed by a sudden expansion into the combustion chamber; the geometric swirl number, $S_{g}$, is 1.3 (computed accordingly to Gupta et al); which is a strong swirl. The flow in the chamber develops into two recirculation zones figure 2. The Outer Recirculation Zone (ORZ) is formed in the wake of the burner exit as a result of the combustor confinement and the Inner Recirculation Zone (IRZ) corresponds to axisymmetric (bubble) vortex breakdown A weak central recirculation region dominated by the exit confinement can also be observed. Full details of the configuration and measurement data can be found in Stopper et al. (2013) and in the TURCHEMI database with its supplemental final report, Stopper (2011a).

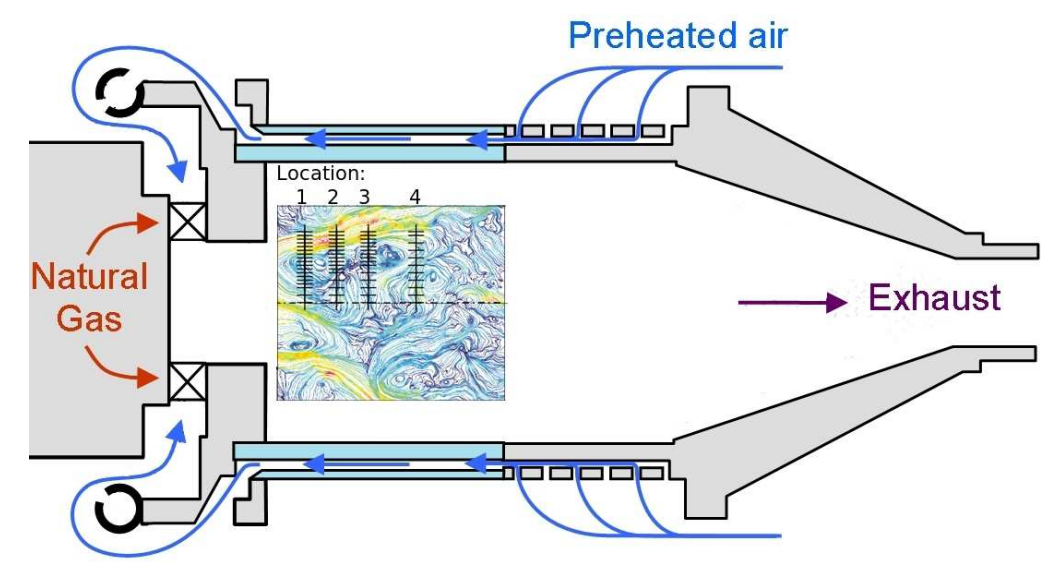

Figure 2: The position of the PIV window and location of $1 D$ Raman measurements in experimental high pressure test rig at DLR Stuttgart for SGT-100 burner, Stopper et al. (2009) 
There were three measurement campaigns, which include PIV, PLIF and 1D Raman and High Speed Imaging. For the non-reactive test case only one testcase at 5 bar was retained and only PIV measurements were considered. Due to limited optical access, only one camera was used and therefore, only the planar flow vectors were determined consisting of the two components in the plane of the laser sheet. More details on the experimental set-up can be found in Stopper (2013) and Stopper (2011b); Stopper et al. (2013).

\section{Computational Details}

The multi-block, structured finite volume BOFFIN-LES code, Jones et al. (2002) has been used for the simulations. It utilises a collocated grid arrangement and second order discretisation schemes are used for all gradients, except for the convective part of the scalar equations for which a TVD-scheme is applied.

The computational domain replicates the actual experimental set-up of the SGT-100 burner studied at DLR Stuttgart, Stopper et al. $(2009,2010)$ without any geometrical simplifications The computational domain consists of the air plenum to the radial swirler, pre-chamber, combustion chamber and an outlet pipe. The overall length of the computational domain is $0.6 \mathrm{~m}$ and the outer diameter of the air inlet is $0.19 \mathrm{~m}$. An additional simulation to investigate the effects of the outlet is performed. The domain was extended to include $0.5 \mathrm{~m}$ of the exhaust pipe of diameter $0.1 \mathrm{~m}$. This simulation captures more of the experimental rig Stopper (2011a) but does not include the exit nozzle located downstream of the exhaust pipe. 


\subsection{Boundary Conditions}

Boundary conditions in the LES were set for the air inlet, a panel air inlet (experimental leakage) and the outlet. The computational domain starts upstream the swirler vanes where air inlet conditions are defined. The computational domain ends at the exit of the combustor exit duct. The air inlet is $2 \mathrm{~mm}$ inwards after the perforated plate used in experiments. The Reynolds number was based on the air inlet diameter of $0.19 \mathrm{~m}$ for all cases discussed herein. The air was introduced in the LES domain via two inlets: main combustion air and panel air (leakage) flow. Fuel was not included in isothermal calculations. All walls were treated with a log-law wall function, Piomelli and Balaras (2002) and a single outlet was used. Inflow boundaries condition for all air inlets are applied as constant uniform inlet velocities. For the main combustion air, a normal velocity component was applied to cell faces across the circular inlet boundary surface and for the air leakage flow an axial component only was specified as the inlet surface is normal to the $x$-axis of the combustor, see figures 3 and 4 . Cell faces from a 2-cell wide perimeter around the combustor were identified and boundary conditions were applied to define the leakage air, as presented in figure 3 and figure 4 . The velocity value was calculated from the mass flow and density used in the experiment and corresponding inlet area. Inlet boundary conditions were set for all boundaries without the addition of any turbulence component. No turbulent component was added at the inlet because the complex flow environment (in particular geometry of the SGT-100 swirler) included in the computational domain will result in high levels turbulence developing as the result of shear layers arising inside computational domain. The radial swirler will generate turbulence in addition to inducing a swirling component to the flow. When instabilities are of interest it is 
common to set domain boundaries to be representative of the acoustic boundaries. In that case, the inlet boundary of the computational domain corresponds to the inlet of air plenum, an enclosed volume prior to the swirler entry. For most of the Preccinsta LES calculations Weigand et al. (2006b) Meier et al. (2007b), the inlet boundary was set at the inlet plenum as a mass flow, Moureau et al. (2011); Franzelli et al. (2012) or as velocity inlet Jones et al. (2012a) for the MERCATO burner. In other LES cases, the computational domain is extended to include the compressor exit, Wolf et al. (2009).

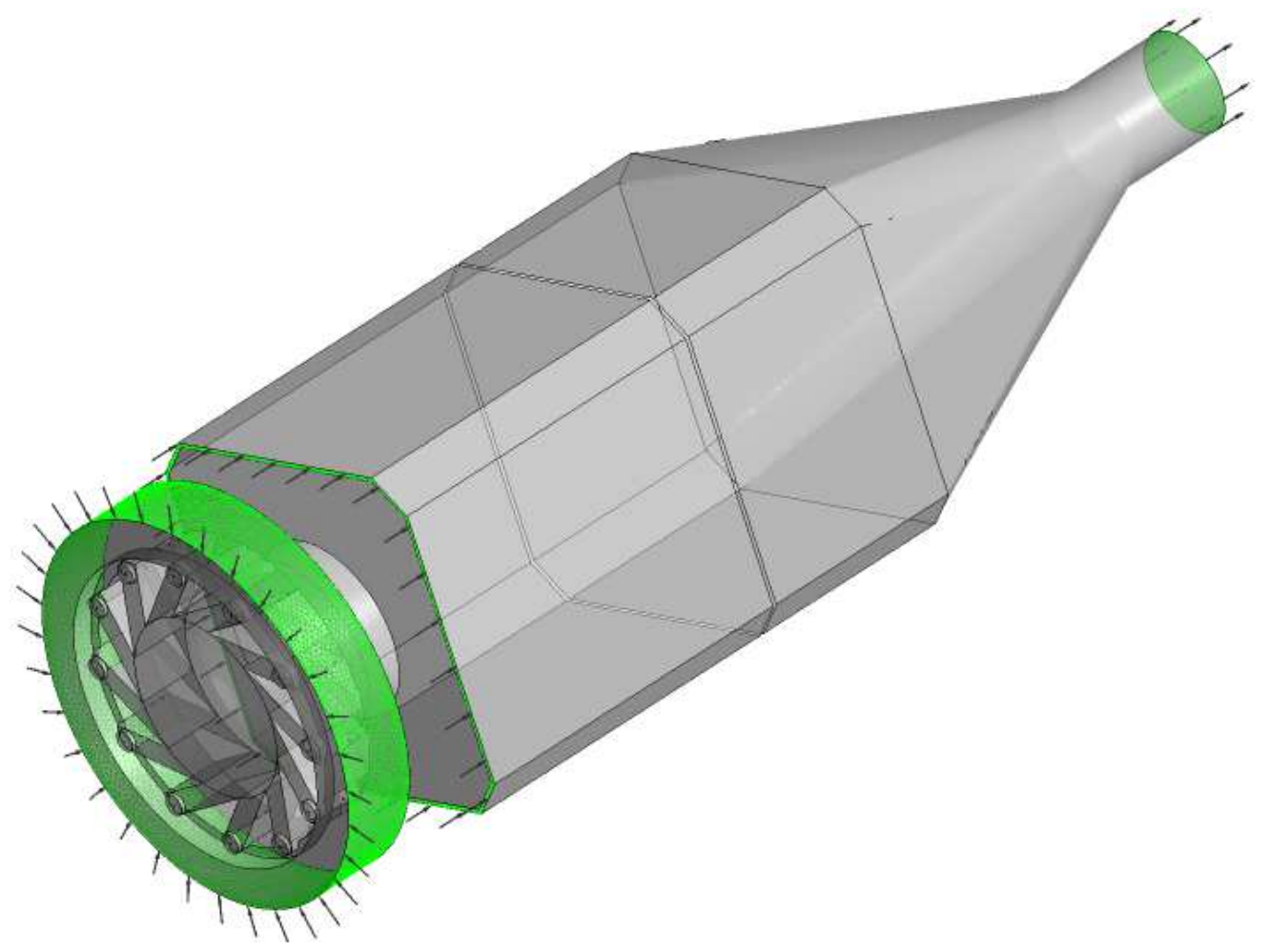

Figure 3: Computational Domain with highlighted air inlet, panel air inlet and outlet surfaces

The specification of outflow boundary conditions in strongly swirling, sub- 


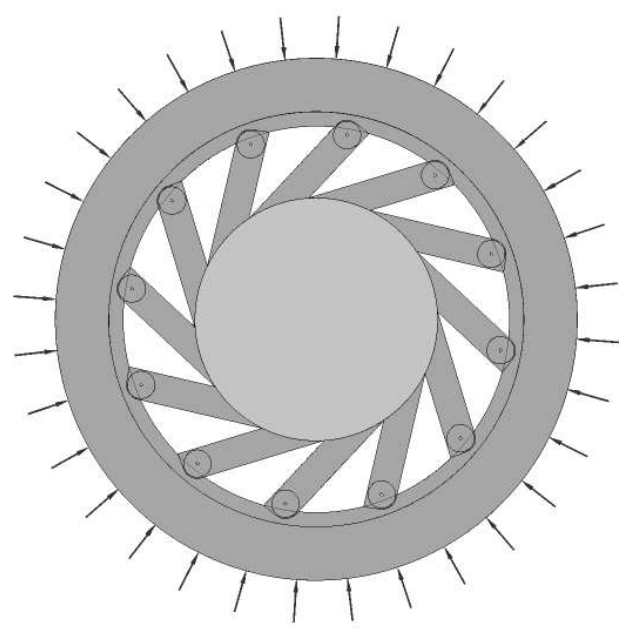

(a) Air Inlet definition

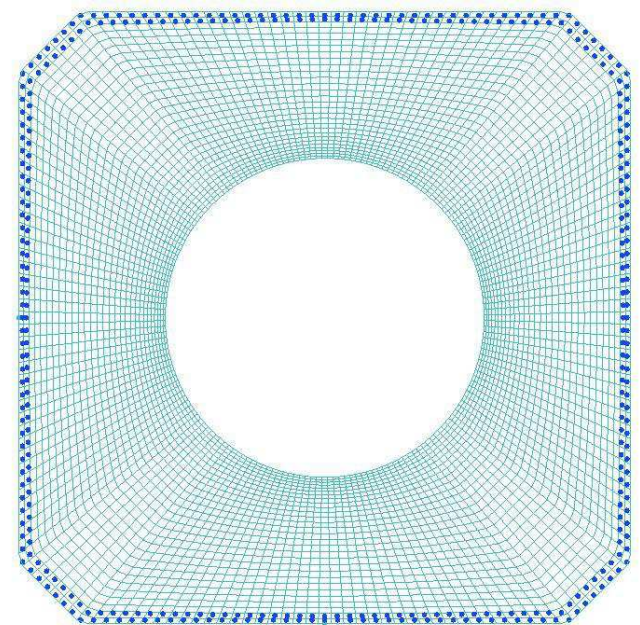

(b) Panel Air Inlet definition

Figure 4: Air inlet definition

critical flows can be problematic. Commonly used outflow conditions include $(i)$ a specified constant pressure together with zero gradient conditions for the velocity components tangential to the outlet plane and other variables, (ii) a convective outflow condition, Le et al. (1997) and (iii) zero gradient conditions applied to all variables, applied so as to allow (strong) vortices to be convected out of the domain. The first of these is inappropriate in strongly swirling flows where swirling motion is usually present at the outflow plane and, while a convective outflow condition allows turbulent structures to be smoothly convected out of the domain, a suitable convective velocity is particularly difficult to define in the presence of recirculation near the outlet, Clayton (2006). For this reason in this work a zerogradient outflow boundary condition was applied at all outflow surfaces.

In previous LES studies, the computational domain was extended to capture the exit flow conditions. For example, Franzelli et al. (2012) extended the com- 
putational domain downstream of the combustion exit so that a part of the outside atmosphere is included. Another option is to simulate the actual exit boundary such as a set of NGVs, Roux et al. (2008). In the majority of computations carried out in the present study the solution domain extended to include the combustor nozzle with the outlet plane coinciding with the nozzle exit. It was found that the $\mathrm{CVC}$ extended throughout the combustor and was evident close to the outflow plane. For this reason and in an attempt to determine the influence of the outlet conditions on the solutions the length of the solution domain was increased to include a portion of the exit pipe.

As full near-wall resolution of the flow is not practical for high Reynolds number flows, and despite flow separations from the swirler, pre-chamber and combustion chamber walls, a semi-logarithmic wall law is applied to all solid boundaries, Piomelli and Balaras (2002). For the swirler, pre-chamber and combustor the first grid points from the wall are positioned at an averaged wall-normal distance of approximately $y^{+}=341, y^{+}=110$ and $y^{+}=75.4$ for Grid I, II and III respectively. Turbulent production from the walls is, however, considered small compared to that generated from geometrical constraints of the swirler and recirculation boundaries within the combustor.

\subsection{Grid}

The minimum and maximum cell spacing in $x, y$ and $z$ directions are presented in Table 1. The cell minimum spacing is located inside the swirler vanes, whilst the maximum cell spacing is located inside the combustor. Grid I was used as the starting point for subsequent grids and most of isothermal LES runs were completed for this grid. The mesh redistribution for Grid II as well as the mesh refinement, Grid III was completed in the downstream part of the combustor in the 
axial $(x)$ direction. This was done in an attempt to better capture the exit core and its interaction with the flow in the front part of the chamber. The cell distribution indicates that the majority of cells is smaller than $2 \mathrm{~mm}$ with very few cells larger.

Table 1: LES cases investigated

\begin{tabular}{|ccccc|}
\hline & Grid I & Grid II & Grid III & Grid IIe \\
\hline Blocks & 240 & 240 & 254 & 256 \\
Mesh Size & 6728307 & 6728307 & 8057282 & 8013165 \\
$\Delta x_{\min }(m m)$ & 0.283 & 0.283 & 0.283 & 0.283 \\
$\Delta x_{\max }(m m)$ & 4.14 & 4.1 & 2.43 & 4.1 \\
$\Delta y_{\min }(m m)$ & 0.0859 & 0.0859 & 0.0859 & 0.0859 \\
$\Delta y_{\max }(m m)$ & 5.29 & 5.27 & 5.02 & 5.27 \\
$\Delta z_{\min }(m m)$ & 0.0859 & 0.0859 & 0.0859 & 0.0859 \\
$\Delta z_{\max }(m m)$ & 5.27 & 5.27 & 5.07 & 5.27 \\
\hline
\end{tabular}

\subsection{Simulation Cases}

Several LES test cases were completed to investigate the influence of the subgrid turbulence model and the influence of local mesh redistribution and mesh refinement; the basic details are summarised in Table 2 .

Case I - the 5 bar pressure case $(\mathrm{Re}=169510)$ using constant Smagorinsky values of $C_{s}=0.1$, without mesh refinement. This case was based on the developing flow inside the combustor, whilst the contraction and exit of the combustor was not fully resolved. In this way the flow in the front of the combustor was developed without the influence (if any) of the flow from the exit of the combustor. 
The bulk average velocity was $9 \mathrm{~m} / \mathrm{s}$ resulting in a Reynolds number, based on the inlet diameter, of 169,510 .

Case II - is identical to Case I except that the mesh has been rearranged in an attempt to provide adequate resolution over the entire computational domain. This was used as a reference case for the operating pressure of 5 bar for comparison with subsequent cases.

Case III - the effect of local mesh re-distribution inside the combustion chamber. This case is identical to Case II but with a different grid distribution.

Case IV - the effect of dynamic Smagorinsky model, Piomelli and Liu (1995). This case is identical to Case III with the dynamic Smagorinsky sgs model being used.

Case IVe - Same as Case IV but with extended domain.

Case V - the effect of mesh refinement inside the combustion chamber, particularly towards the exit. This case is identical to Case IV except that a more refined mesh is used.

Cases VI and VII are identical to case II except that the operating conditions are varied to give different Reynolds numbers. For both cases the bulk averaged velocity was increased to $25 \mathrm{~m} / \mathrm{s}$ and the operating pressure was reduced to 3 bar for Case VI and increased to 6 bar for case VII. This resulted in Reynolds numbers 30/conditions and the same Grid I was used for both cases. There are no experimental data available for these two cases.

All the simulations to be presented have been completed with a constant time step of $\Delta t=5 \times 10^{-7} \mathrm{~s}$ ensuring a maximum Courant number not greater than 0.35. This value of Courant number was selected to ensure temporal accuracy, whilst a constant time step is needed for FFT. It should be noted that the same 
Table 2: LES cases investigated

\begin{tabular}{|ccccc|}
\hline & Grid size & Grid Type & Dynamic Model & $R e$ \\
\hline Case I & $6.7 \mathrm{M}$ & I & $\mathrm{N}$ & 169510 \\
Case II & $6.7 \mathrm{M}$ & II & $\mathrm{N}$ & 169510 \\
Case III & $6.7 \mathrm{M}$ & II & $\mathrm{N}$ & 169510 \\
Case IV & $6.7 \mathrm{M}$ & II & $\mathrm{Y}$ & 169510 \\
Case IVe & $8.0 \mathrm{M}$ & IIe & $\mathrm{Y}$ & 169510 \\
Case V & $8.1 \mathrm{M}$ & III & $\mathrm{Y}$ & 169510 \\
Case VI & $6.7 \mathrm{M}$ & $\mathrm{I}$ & $\mathrm{N}$ & 118810 \\
Case VII & $6.7 \mathrm{M}$ & $\mathrm{I}$ & $\mathrm{N}$ & 237714 \\
\hline
\end{tabular}

boundary conditions were maintained between all the LES test cases. Due to the recirculating nature of the flow field a meaningful flow through time is difficult to define. For the reference case, the burner reference time scale based on the bulk velocity $\left(U_{b}=9 \mathrm{~m} / \mathrm{s}\right)$ is $t_{b}=12 \mathrm{~ms}$. In case of increased/decreased Reynolds number (Case VI and Case VII) the burner reference time scale based on the bulk velocity $\left(U_{b}=25 \mathrm{~m} / \mathrm{s}\right.$ is $t_{b}=4.55 \mathrm{~ms}$ The recirculation period of the slowest recirculation zone or PVC structure may however represent a more representative characteristic time. The flow development period for the reference case was 173.2 $\mathrm{ms}$, which is equivalent to $27 \mathrm{PVC}$ cycles (one PVC cycle corresponds to 6.33 $\mathrm{ms}$ ). During parametric analysis it important to allow the simulation to become 'fully developed' before collecting statistics; a development period of at least 100 ms was applied to all cases.

Turbulent scales for the three cases with differing Reynolds numbers are pre- 
sented in Table 3. The Kolmogorov scales can be estimated given the magnitude of the large eddy scales. The Kolmogorov length $L_{K}$, time $\eta_{K}$ and velocity $v_{K}$ scales were estimated based on the large eddy length scale $L_{t u r b}$, which was taken to be the pre-chamber diameter $D_{p r}=0.086 \mathrm{~m}$ as being representative of the large scale turbulent motion. The velocity scale $v^{\prime}$ was estimated from the velocity at the pre-chamber exit. The Kolmogorov length $\eta_{K}$ scales are of two orders of magnitude lower than the mesh sizes.

Table 3: Turbulent scales of cases investigated

\begin{tabular}{|cccc|}
\hline & $R e$ & $v^{\prime}$ & $\eta_{K}(\mathrm{~mm})$ \\
\hline Case I -IVe & 169510 & 5 & 0.00852 \\
Case VI & 118111 & 13.8 & 0.0112 \\
Case VII & 237714 & 13.8 & 0.0663 \\
\hline
\end{tabular}

\section{Results}

The main results for Case II are taken as the reference and comparative analyses were performed in relation to this case. Results are presented and compared in form of $1 \mathrm{D}$ profiles of mean and RMS values of axial and radial velocity components. The PIV measurement data is available only for two velocity components. Firstly, detailed comparison between time-averaged LES results and PIV measurements is presented and discussed for the reference case. Then main results of several further computations are presented. 


\subsection{Reference Case}

An instantaneous snapshot of the velocity field throughout the combustion chamber is presented in figure 5. Two main recirculation regions are observed: inner and outer, which are discussed later in this section. The resolved kinetic energy is presented in figure 6 as a slice through the swirler and a slice though the combustion chamber. High levels of the kinetic energy are generated by the swirler device. In the combustion chamber, most of the kinetic energy is generated inside the pre-chamber and in the front of the combustor, where the mesh quality is good. This supports the choice of inlet boundary conditions without the addition

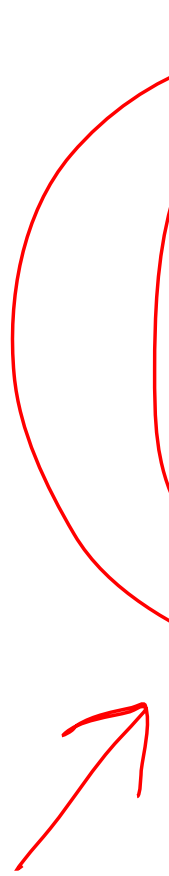
of synthetic turbulence

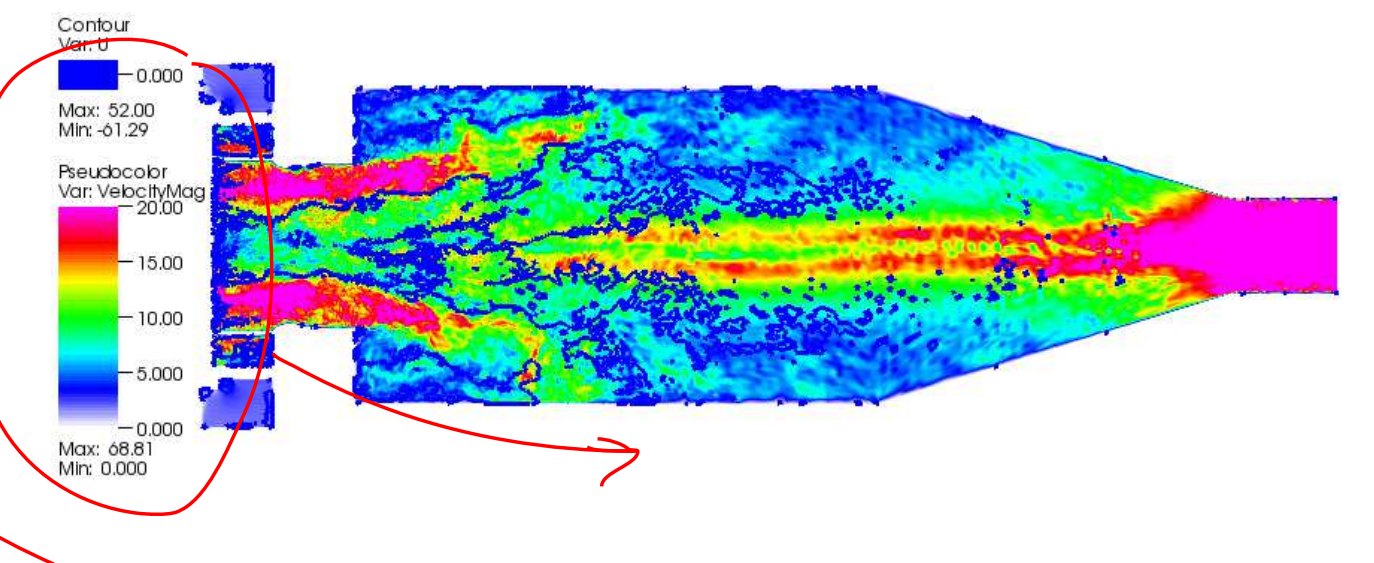

Figure 5: A snapshot of velocity field $(\mathrm{m} / \mathrm{s})$ through the SGT-100 combustor with $\mathrm{U}=0$ line to distinguish recirculation regions.

An iso-contour of low pressure, presented in figure 7, depicts coherent structures as suggested by Poinsot and Veynante (2005) and Wang et al. (2007). Isocontours are shown at four different angles around the axis of rotation, measured from an arbitrary datum. Two separate structures are observed: a Precessing Vortex Core (PVC) and a Central Vortex Core (CVC), The two coherent flow features 


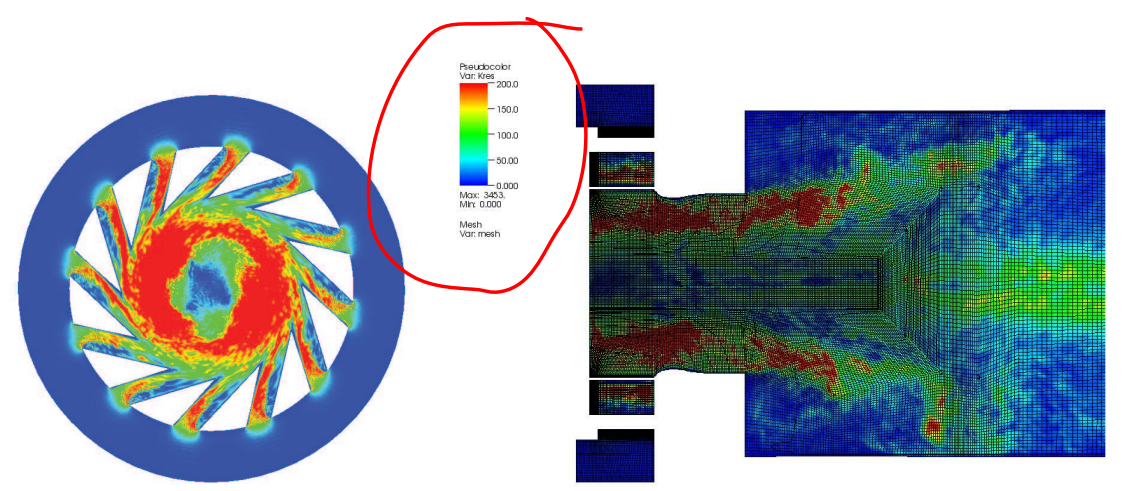

(a) Slice through the swirler.

(b) Front of the combustor

Figure 6: A snapshot of the resolved turbulent kinetic energy in the swirler and in the front of the combustor.

are visible in as three-dimensional vortex structures with long lifetimes. The PVC precesses along the shear layer around the combustor centre axis in a tangential direction introduced by the swirl.

Occasionally, a second out-of-phase component of the PVC is formed in its wake, leading to a double helical structure of the PVC, which may also be seen on figure 7 at $270^{\circ}$. However, the second helical structure is weaker than the first and most of the time the PVC consists of a single helical structure. The CVC is formed at the tail end of the Inner Recirculation Zone and is precessing on the centre axis in the same direction as the PVC. Occasionally, the CVC may attach to the pilot face at $180^{\circ}$.

The temporal auto-correlation coefficient of the axial velocity was normalised to identify the main frequencies of the flow field. Data sampled at $2 \mathrm{MHz}$ from two points shown as a log-log plot in figure 8 . The time series data is in the form of an energy density spectrum of the auto-correlation coefficient using a 


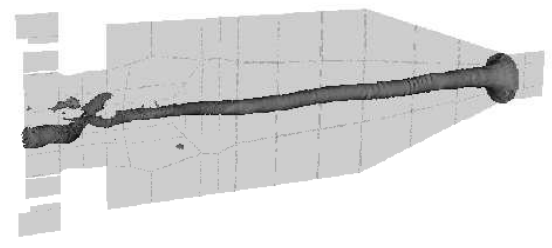

(a) $0^{\circ}$

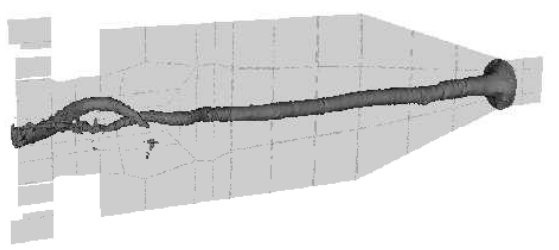

(c) $180^{\circ}$

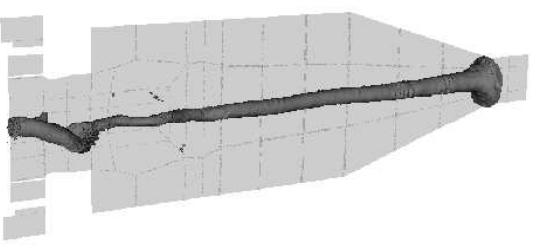

(b) $90^{\circ}$

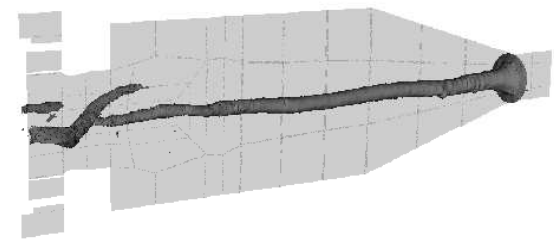

(d) $270^{\circ}$

Figure 7: Coherent structures inside the combustor, PVC and CVC presented as iso-contour of low pressure.

Hamming window. Point $100^{1}$, located on the centre axis, does not indicate any dominant frequency, whilst the Point 113 , located in the inner shear layer, shows the frequency of the PVC of $158 \mathrm{~Hz}$. Based on the vortex radius of 0.01 $\mathrm{m}$ that forms the PVC and the main flow velocity, the rotational frequency can be estimated to be $159 \mathrm{~Hz}$. For both points a minor peak of approximately $83 \mathrm{~Hz}$ is observed, a similar magnitude to the PVC frequency. This is associated with the axial flow decay into the combustion chamber at the frequency related to the burner residence time of $12 \mathrm{~ms}$. Although the location of the CVC was identified, its dominant frequency was not captured, because its strength was poorly captured by LES. Some attempts to improve CVC predictions by the LES are presented

\footnotetext{
${ }^{1}$ The numbering and the location of the points is given in Stopper et al. (2013)
} 
later in the paper.

The line for $k^{-5 / 3}$ was included to determine the range of energy decay corresponding to inertial range. Most of the energy is contained in a low frequency range with the inertial range starting above $5000 \mathrm{~Hz}$.

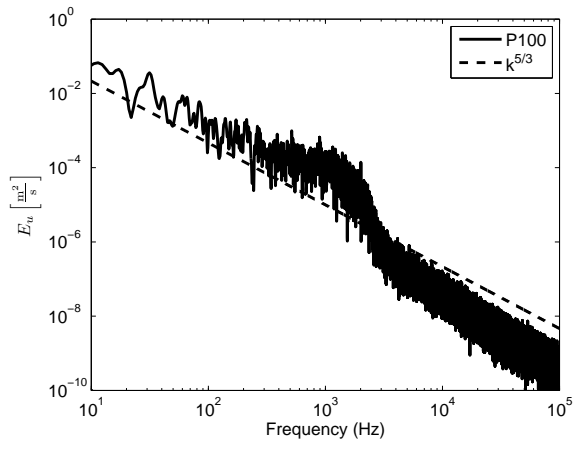

(a) Point 100 on centre axis

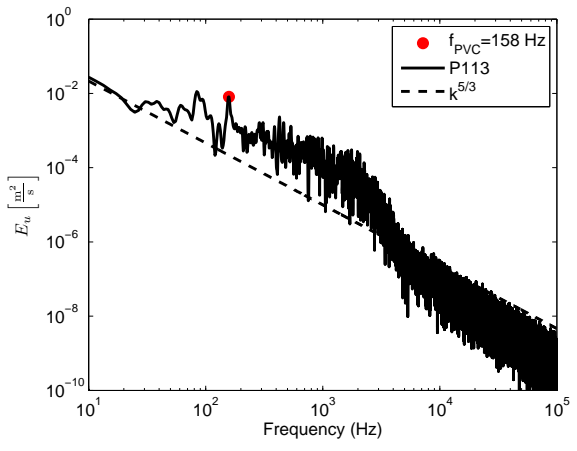

(b) Point 113 in the shear layer

Figure 8: Energy Density Spectrum of the auto correlation coefficient of the axial velocity.

Figure 9 displays the comparison between mean and RMS LES results and PIV measurements at four axial locations $(x / D=1.21,1.44,1.66,2.00)$. The simulated profiles of both axial and radial velocities are, in general, in good agreement with experimental data in terms of the location and magnitude of the maxima and minima. The location of all three shear layers is well captured by the LES. The magnitude of the axial velocity and the RMS of its fluctuations on the centre axis is under-predicted by the simulation. However, the magnitude of both inner and outer shear layers were captured very well at all locations. The radial velocity was also well predicted for both mean and RMS values. Again the centre axis was slightly under-predicted, particularly for the RMS fluctuations. In general, the RMS values were observed to be higher on the centre axis indicating that a 
strong instability (such as CVC) is present in this region.

Figure 10 shows a comparison of contour plots of mean (left) and RMS (right) of the axial velocity from the LES results and the PIV measurements. For ease of comparison, the same colour scheme and the same scale were used to generate all plots. A line of $U=0$ was included to indicate the location and size of vortex breakdown. The results of the simulations presented on top of figure 10 depict the main flow structures such as the outer recirculation zone, vortex breakdown and give an indication of the location of the shear layer regions. Excellent agreement between the simulation and measurements can be observed when the LES result are overlayed by contours of the PIV measurements, figure 10(bottom). From this comparison is easy to conclude that the size and magnitude of the CVC is under represented by the simulation.

Figure 11 shows a comparison of the radial velocity from the LES and the PIV measurements. The format of figure 11 is similar to figure 10 and supports the good agreement between simulation and measurements. The simulated radial velocity under estimates the flow structure corresponding to CVC, also referred to as the exit core. The flow from the exit core changes direction from positive to negative in the LES, whilst in the PIV measurement it is always negative. This may be associated with the strength of the exit core that is under represented the LES and thus changes its rotation at the tail end. It is also possible that this is also related to the limited interrogation window of the PIV and the change in flow direction occurs later in the experiment.

The axial and radial velocity components from both LES and PIV were used to generate the absolute planar velocity fields. Vector fields of the absolute planar velocity were also generated to compare the flow direction of the main flow 

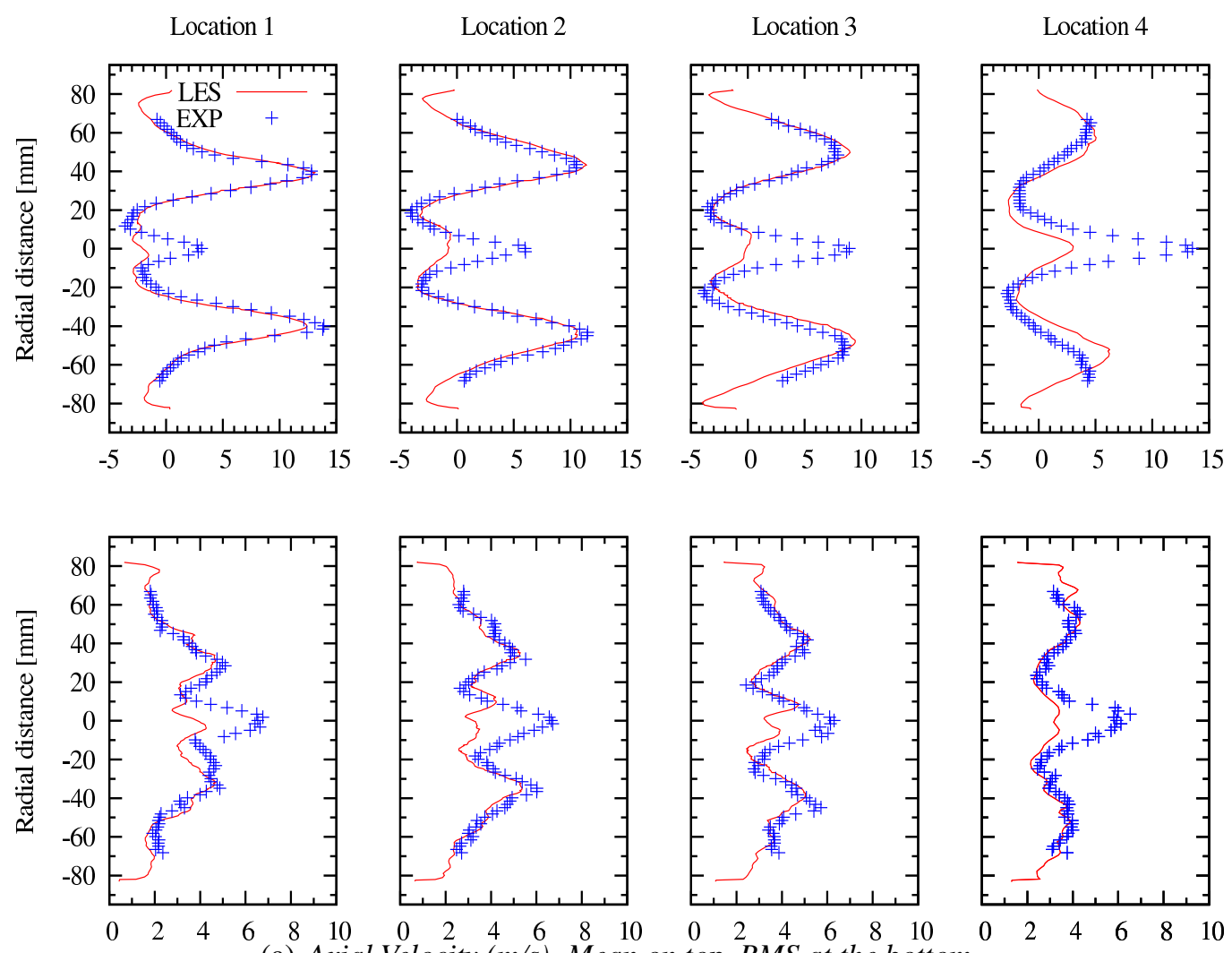

Location 1

Location 2

Location 3

Location 4
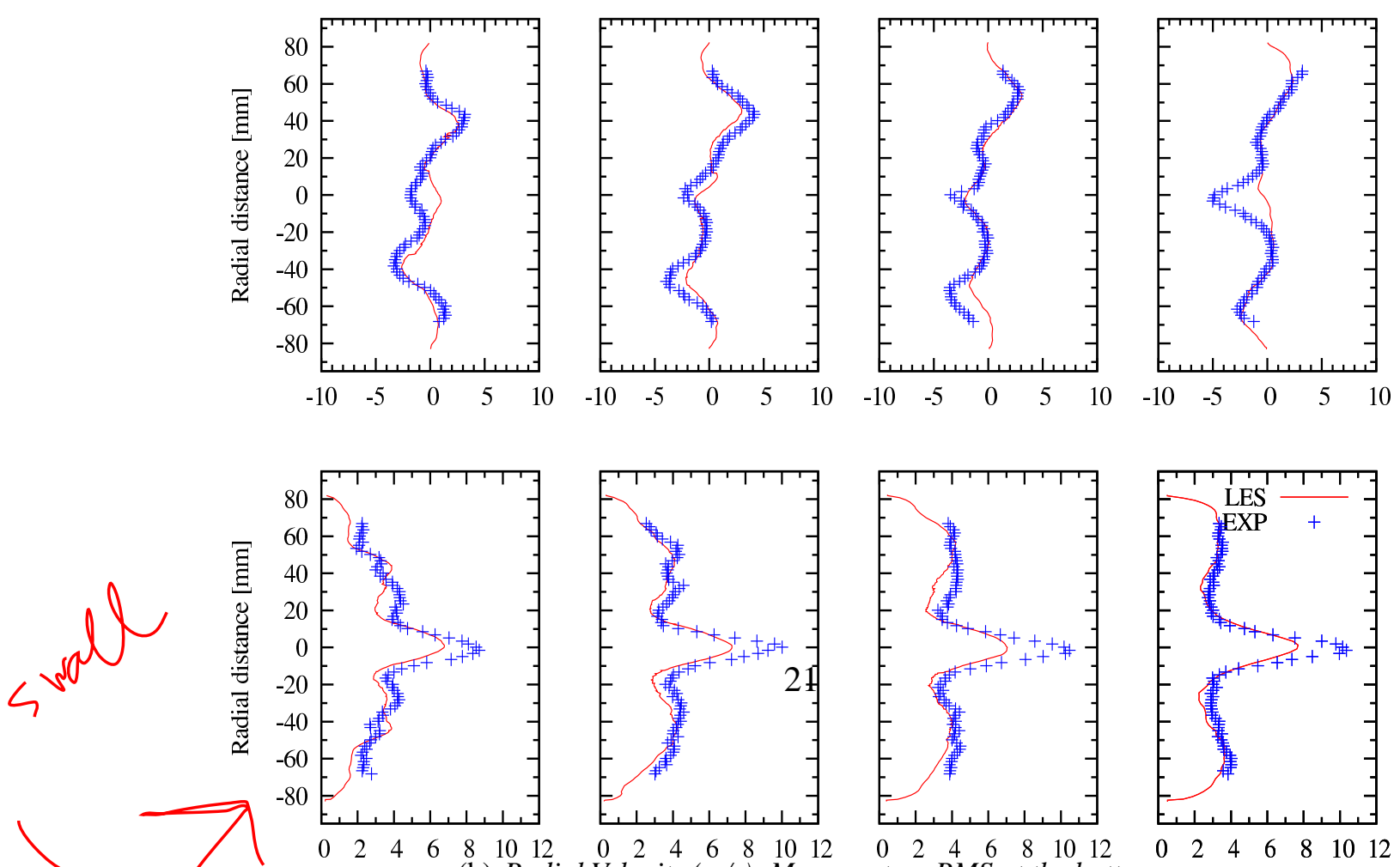

(b) Radial Velocity $(\mathrm{m} / \mathrm{s})$. Mean on top, $R M S$ at the bottom.

Figure 9: The gomparison between LES and Experiment from Stopper (2011a); the locations are as given in figure 2 

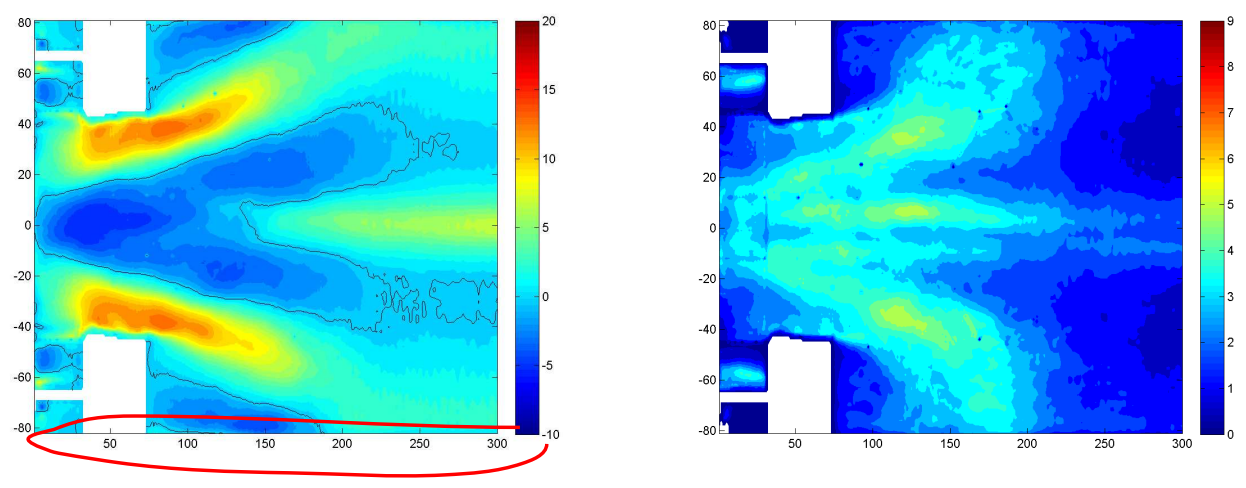

(a) LES results
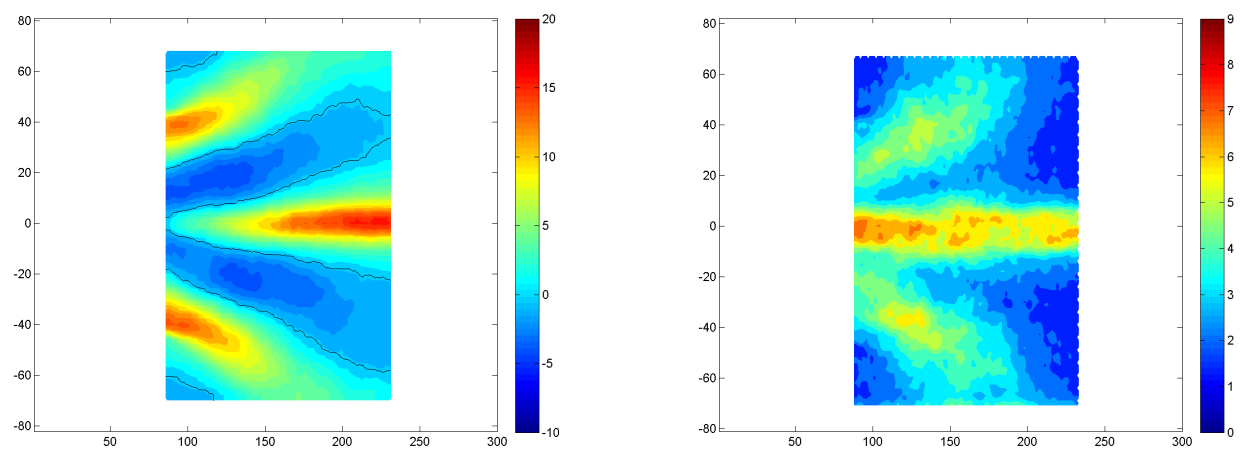

(b) PIV measurement
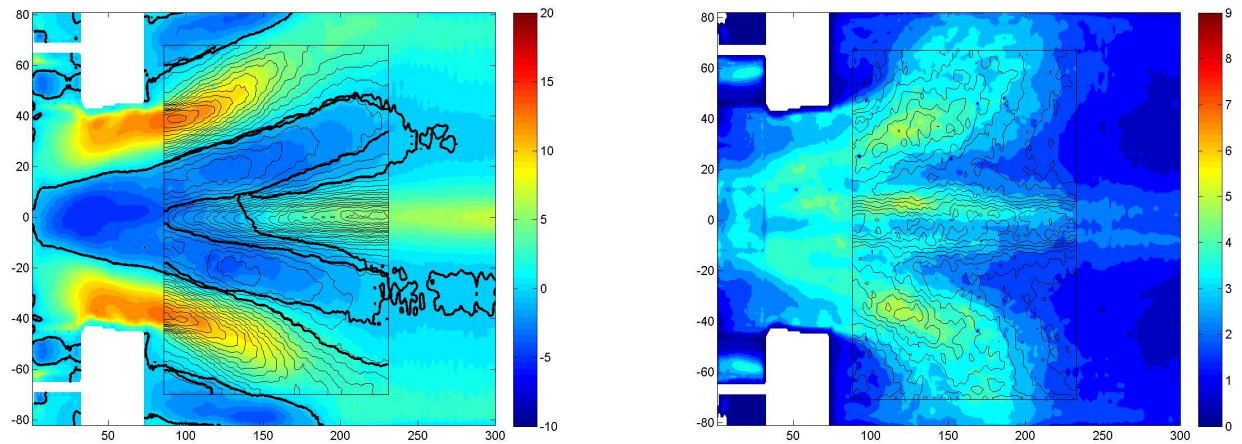

(c) LES with overlayed PIV

Figure 10: The comparison between LES and Experiment axial velocity: means (left) and RMS (right). 

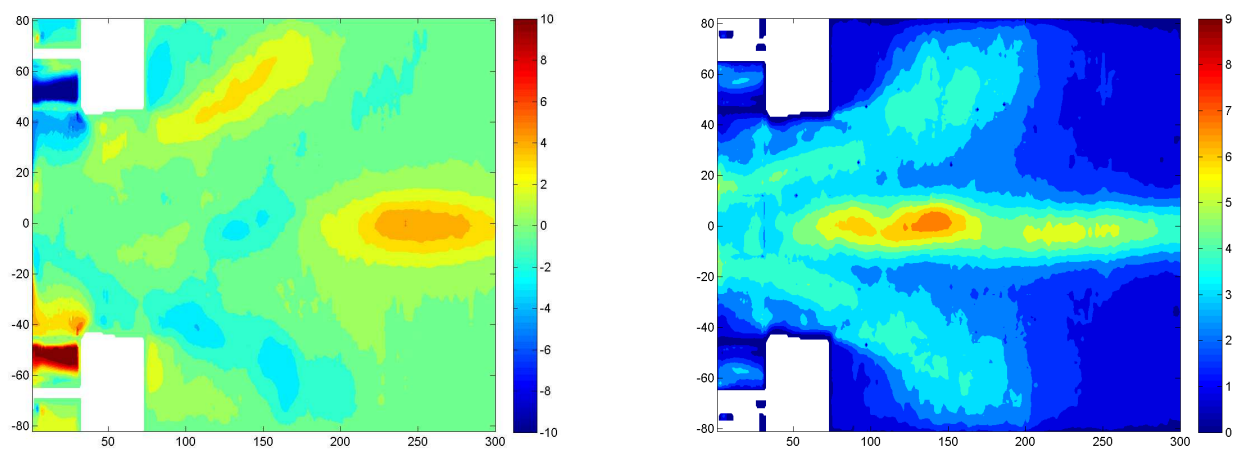

(a) LES results
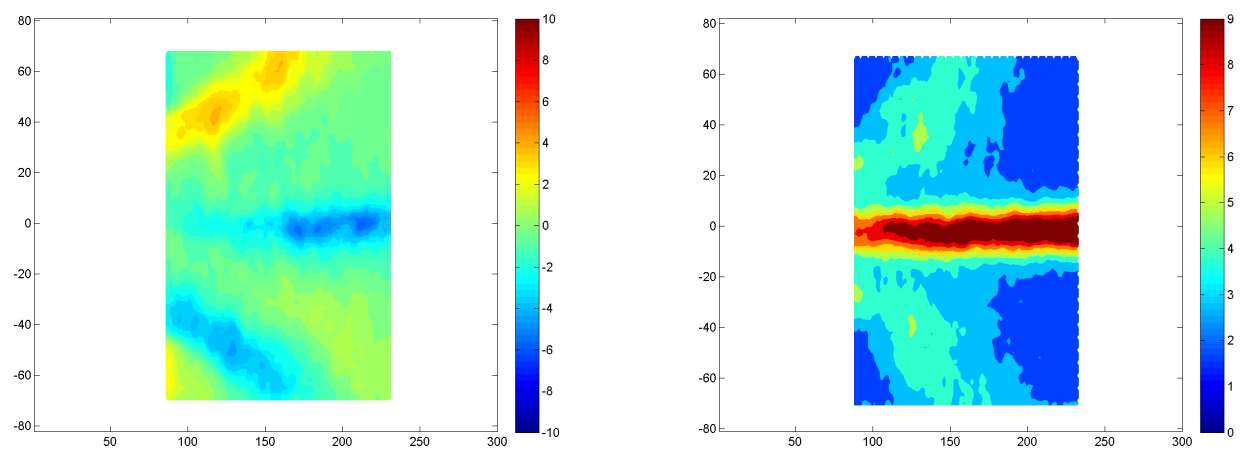

(b) PIV measurement
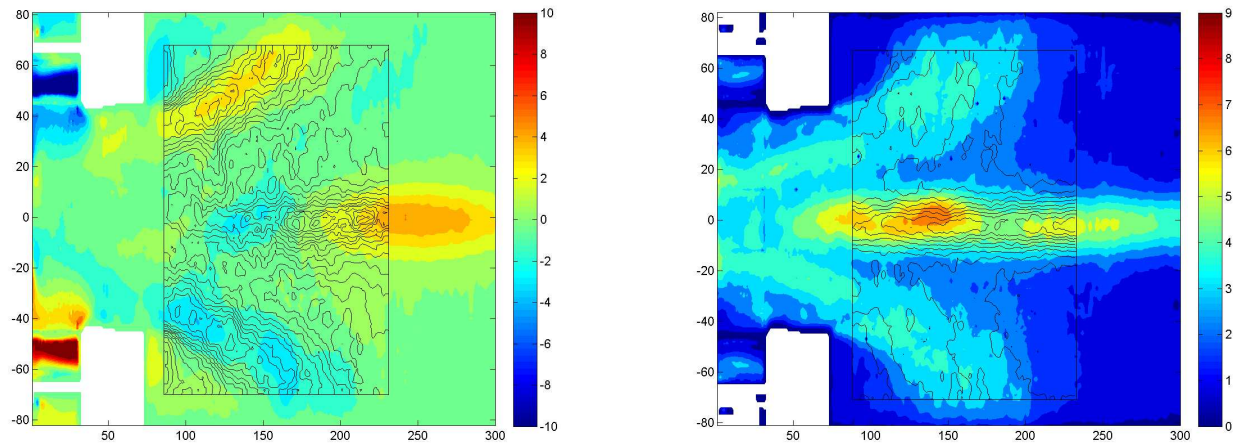

(c) LES with overlayed PIV

Figure 11: The comparison between LES and Experiment radial velocity: means (left) and RMS (right). 
structures. A comparison between the LES results and the PIV measurements for the absolute planar velocity and vectors fields is presented in figure 12. The direction of the vector fields and the flow path is well reproduced. From the swirler vane exit, the air passes through the pre-chamber and then enters the combustor chamber. Inside the pre-chamber, the swirl component is strong and thus most of the forward flow is pushed outward, closer to the pre-chamber wall. After the sudden expansion, the axial momentum of the swirling flow suddenly decays and under the influence of a strong radial pressure gradient, a part of the flow returns into the front of the burner. The reverse flow into the burner then strongly attaches to the face of the pilot burner where a part of the flow is transported by the PVC to the inner shear layer inside the pre-chamber. Inside the pre-chamber, a second stagnation flow condition forms the tip of the CVC which accelerates the forward flow to the exit. Under swirling conditions, a tangential velocity component is introduced to the flow by the swirl. In addition to the coherent structures and recirculation regions discussed above, highly counter-rotational flows inside the burner and around the shear layers were observed. The vorticity characterises the rotational nature of the flow. In addition to vorticity, the strain rate or rate-ofstrain provides an insight into the deformation of a local volume element by the shear or stretch. As experimental data exist only in 2D, planar vorticity and planar strain rates were evaluated from the simulation in order to determine deformation regions of the fluid.

Figure 13 confirms the strong flow unsteadiness on the face of the pilot burner as well as on the internal wall of the pre-chamber. The shear layers between reverse flows were captured well by the comparison plots of the planar vorticity and strain rate, presented in figure 13 left and right respectively. Furthermore, it 

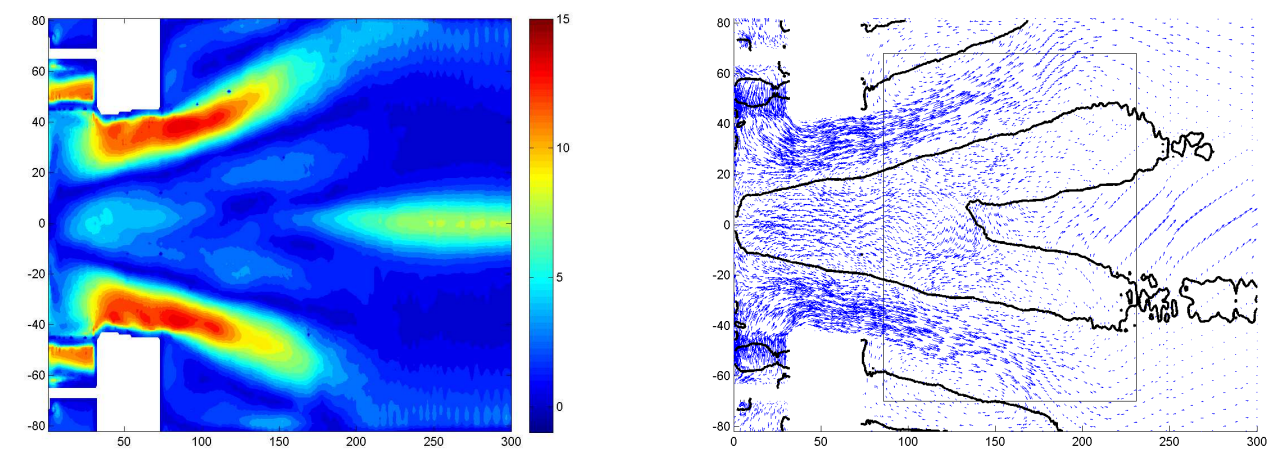

(a) LES results
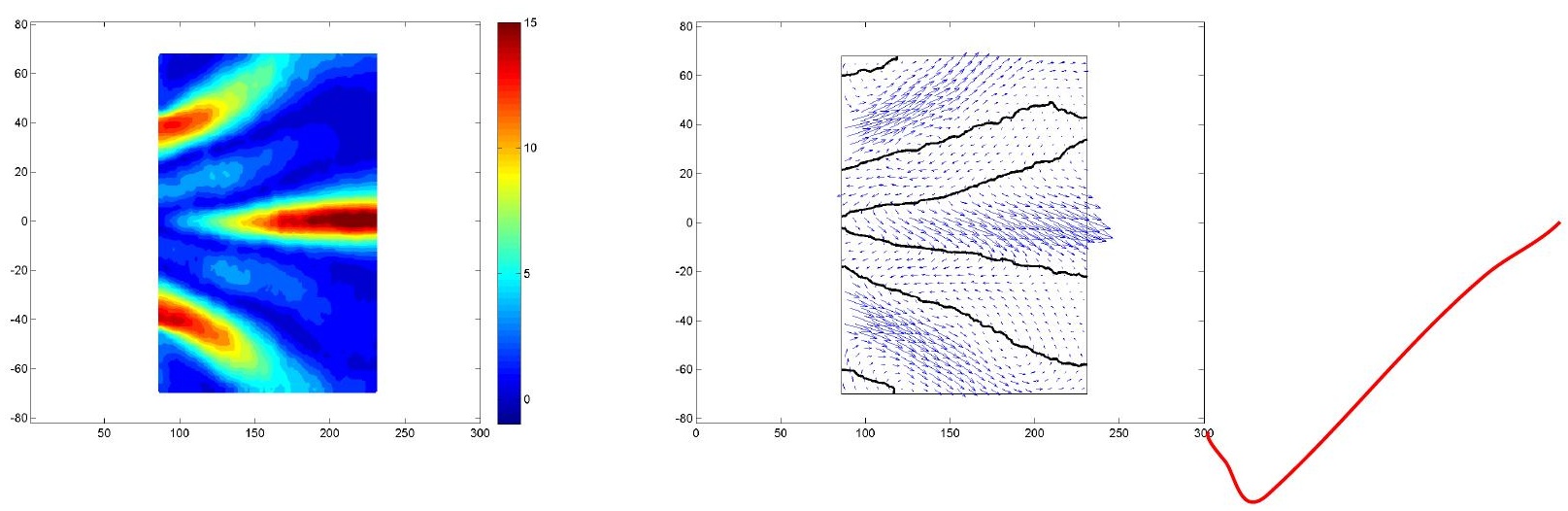

(b) PIV measurement
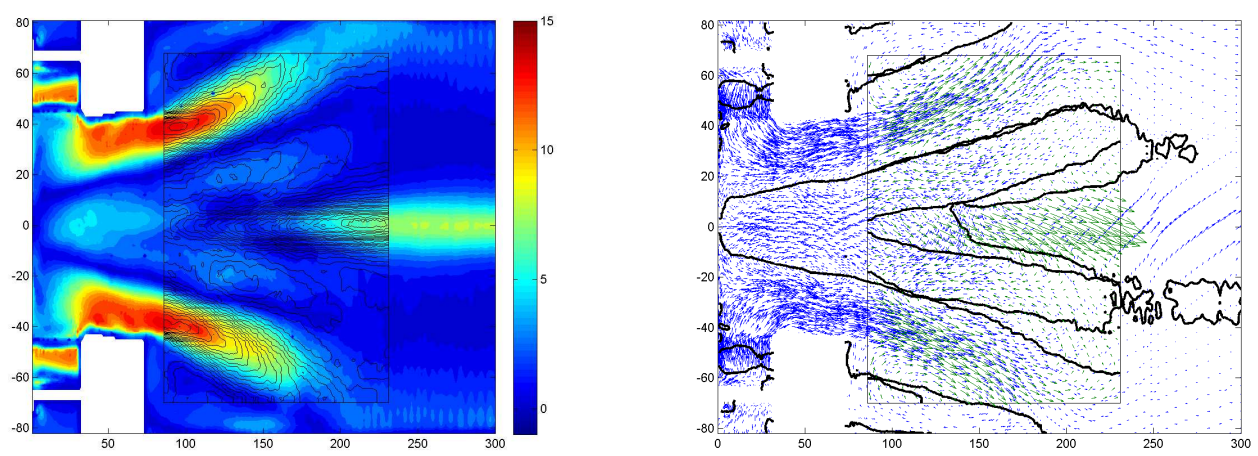

(c) LES with overlayed PIV

Figure 12: The comparison between the isothermal LES and Experiments, absolute planar velocity (left) and planar velocity vectors (right) 
indicates that the rotational rate of the flow direction is opposite in the inner and outer shear layers. The planar strain rate is much higher in the outer shear layer than in the inner shear layer because of slow outer recirculation vortex. Again, the strength of the exit core has not been well reproduced in the simulations

\subsection{Influence of the Dynamic Model}

The sub-grid model may influence the LES results particularly when coarse meshes are used. Also a single turbulent time scale and length scale defined by the Smagorinsky model may not be appropriate in non-isotropic meshes. The sub-grid model will influence the LES results (i) in regimes where the turbulent Reynolds number is low or (ii) in regimes where the mesh resolution is poor. A comparison of the influence of the sub-grid model on the LES results for complex geometries has not been performed previously. Recent simulations of laboratory swirl burners were completed either with constant Smagorinsky Jones et al. (2012a,b); or with dynamic sub-grid model, Jones et al. (2012c). The comparison of LES results from Case III and Case IV using the same Grid II is presented in figure 14. Overall, main flow structures were captured well and the results were not influenced significantly by the choice of sub-grid model. The largest difference in the results arose in the vicinity of the centre axis of the combustor at the first three locations, particularly the mean axial velocity. It suggests that the length of the CVC is not well reproduced by the dynamic model and this is probably due to the poor mesh resolution in the downstream part of the combustor, where the mesh is coarse. Insufficient mesh resolution may result in a progressively larger contribution of the sub-grid dissipation to the turbulent transport, an effect that is likely more pronounced with a dynamic model where the flow must be adequately resolved by the (larger) test filter. In shear layer regions, however, small differ- 

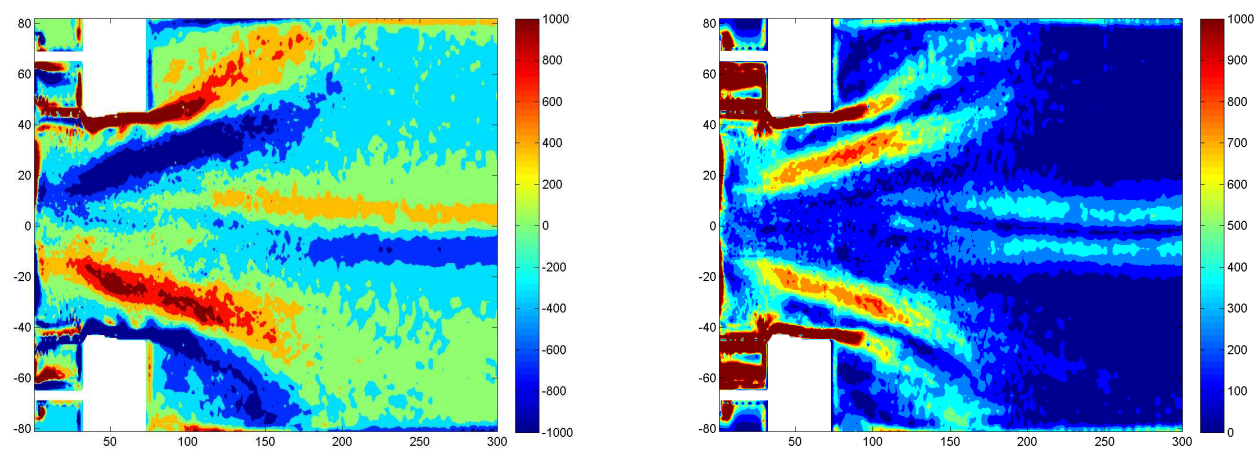

(a) LES results
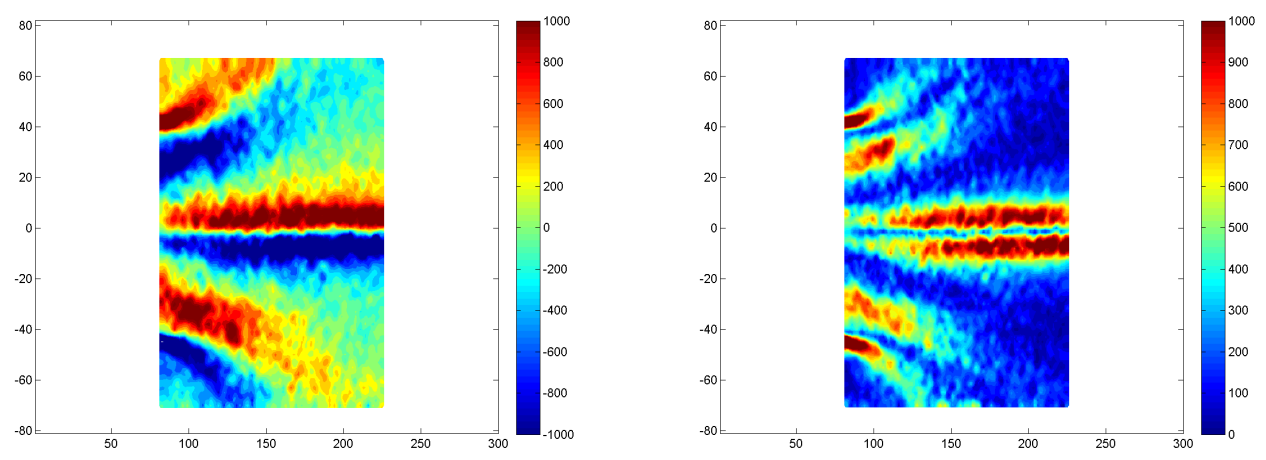

(b) PIV measurement
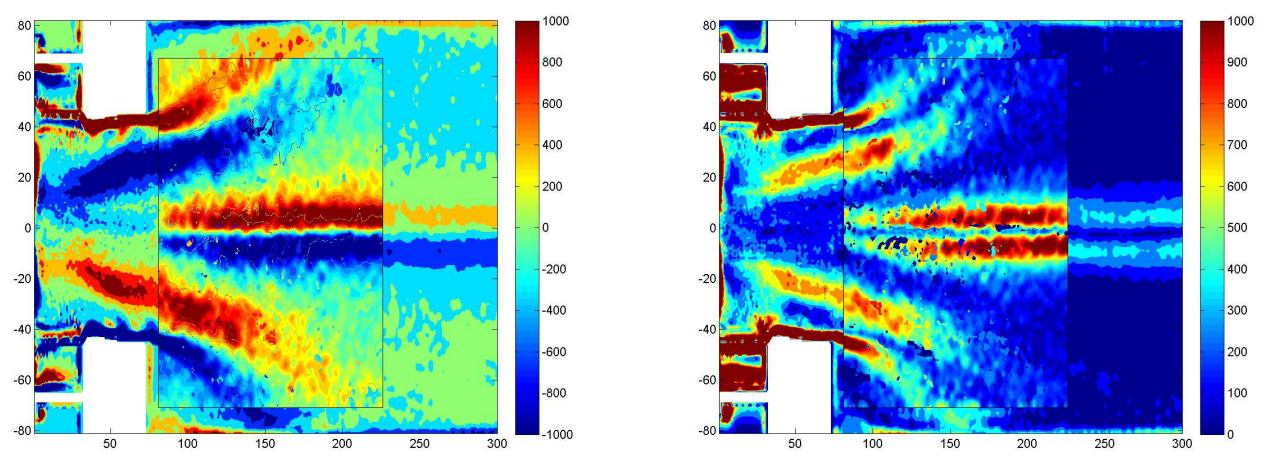

(c) LES with overlayed PIV

Figure 13: The comparison between the isothermal LES and Experiments, planar vorticity (left) and planar strain rate (right). 
ences were observed between both cases. This suggests that the grid resolution at the front of the combustor was adequate. In an adequately resolved LES of high Reynolds number flow, the results are expected to be largely independent of the value of the Smagorinsky constant. and indeed of the precise details of the sub-grid model. The results suggests that the mesh refinement in the downstream part of the combustor may have an influence on the strength of the CVC. This is investigated on in the next section using the dynamic sub-grid model.

\subsection{Mesh Refinement}

The small improvements obtained from the mesh re-distribution from Case III compared to Case II suggest that the mesh quality in the downstream part of the combustor might have an influence on the strength of the exit core and hence a new Grid III, Case V was considered. As the dynamic sub-grid model was used, the results from Case V, were compared with Case IV and are presented in figure 15 . Comparisons of the axial and radial velocity profiles suggest again the solutions are effectively grid-independent. Apart from the exit core, most of the flow structures were captured well with both grids. Despite the doubling of the number of cells in the axial direction and the reduction in cell aspect ratio in the downstream part of the combustor, there was no significant difference between two results. A better flow resolution of the exit core was achieved for the Case $\mathrm{V}$, but the strength of the exit core did not change. Since in all cases the same boundary conditions were used, it is suggested that the strength of the core is associated with poor representation of the exit confinement due to the sub-critical nature of the exit flow. An improved outlet boundary condition may be required to capture the penetration and the magnitude of the CVC. 

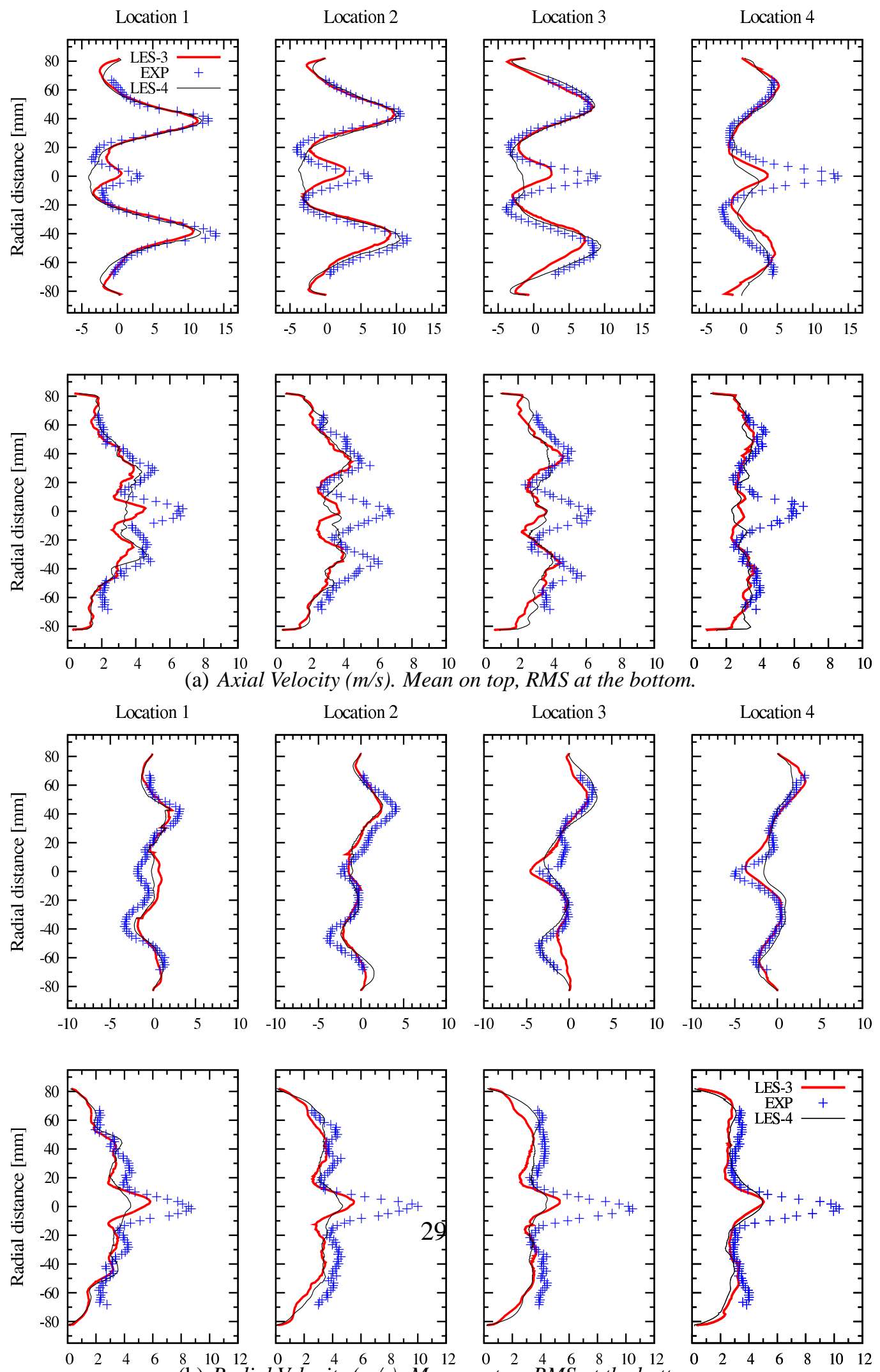

b) Radial Velocity $(\mathrm{m} / \mathrm{s})$. Mean on top, RMS at the bottom.

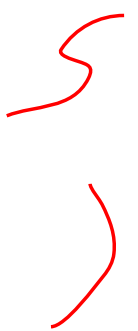

Figure 14: The influence of sub-grid dynamic model. 

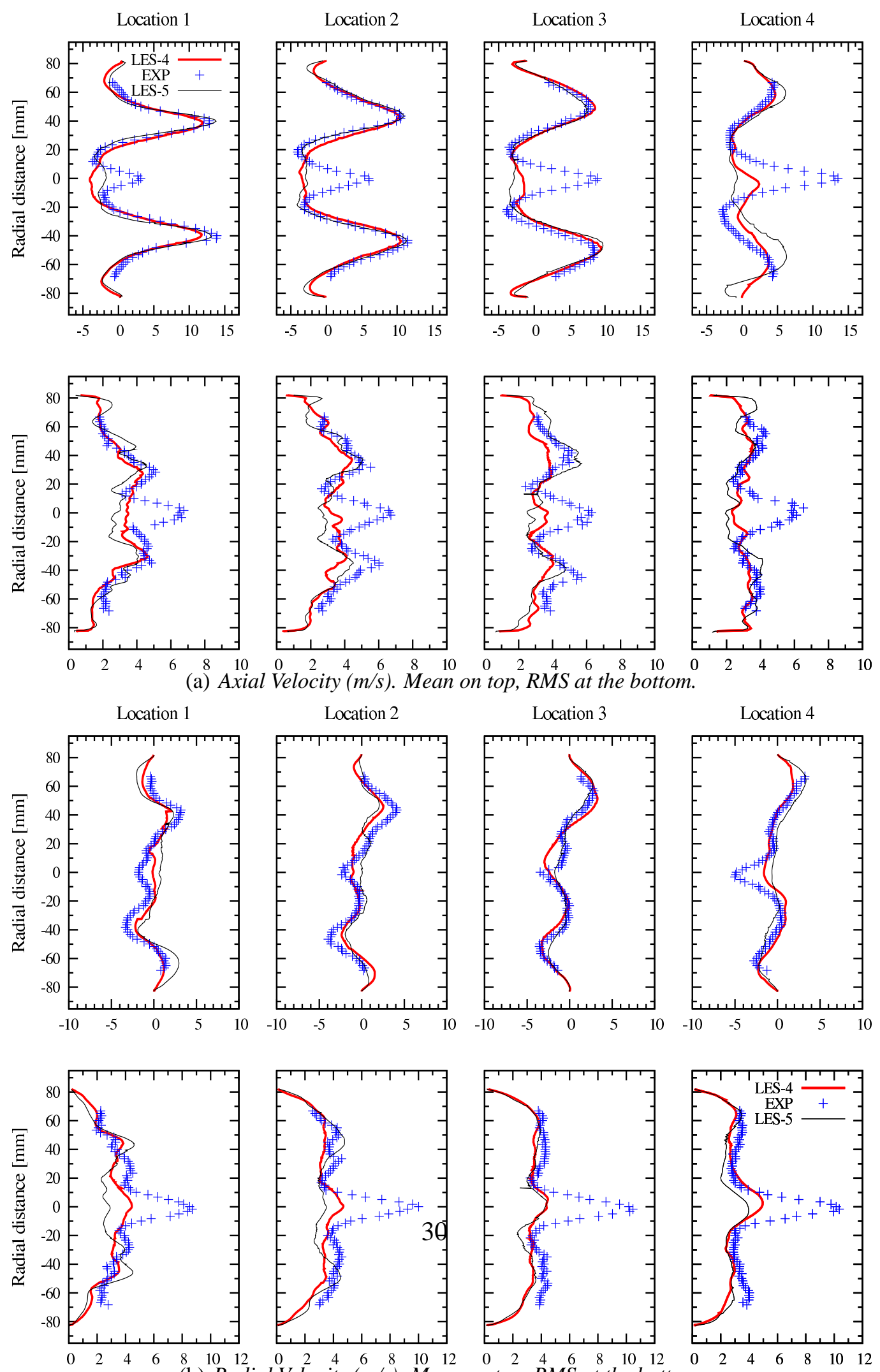

(b) Radial Velocity $(\mathrm{m} / \mathrm{s})$. Mean on top, RMS at the bottom.

Figure 15: The influence of axial mesh refinement. 


\subsection{Extended Solution Domain}

The computational domain was extended to include part of the exhaust pipe. The CVC penetrates the exhaust pipe (see figure 16), although it still remains pseudo attached to the exit of the chamber. The flow expands entering the exhaust pipe, and additional recirculation regions appears. The flow becomes fully developed after approximately $100 \mathrm{~mm}$ and high turbulence is observed. Large pressure fluctuations are present in the pipe as the vortex precess with same frequency and in the main chamber.

The improved outlet description however does not affect the main velocity profiles (figure 17) and the axial velocity remains under-predicted and the central recirculation region is larger than observed in the experiments.

\subsection{Reynolds Number Dependency}

The Reynolds number dependency on the flow structures is presented in this section. Two test cases were considered corresponding to the Reynolds number varying by approximately $30 \%$ either side of that of the reference case. For fully turbulent flows, the solution (in terms of mean and RMS parameters) should be esentially independent of the Reynolds number,Tennekes and Lumley (1972). However, it is possible that the behaviour of some flow aspects such as the PVC or CVC could be affected by a change in the Reynolds number: the smallest scale structures become smaller as the Reynolds number is increased. Unfortunately, there are no experimental data available for the isothermal flows corresponding to Case VI and Case VII. Therefore, the LES results were compared against the scaled LES reference results of Case II. LES results from Case II were scaled by the inlet velocity ratio of 2.76 so that effect of Reynolds number can be quantified. 


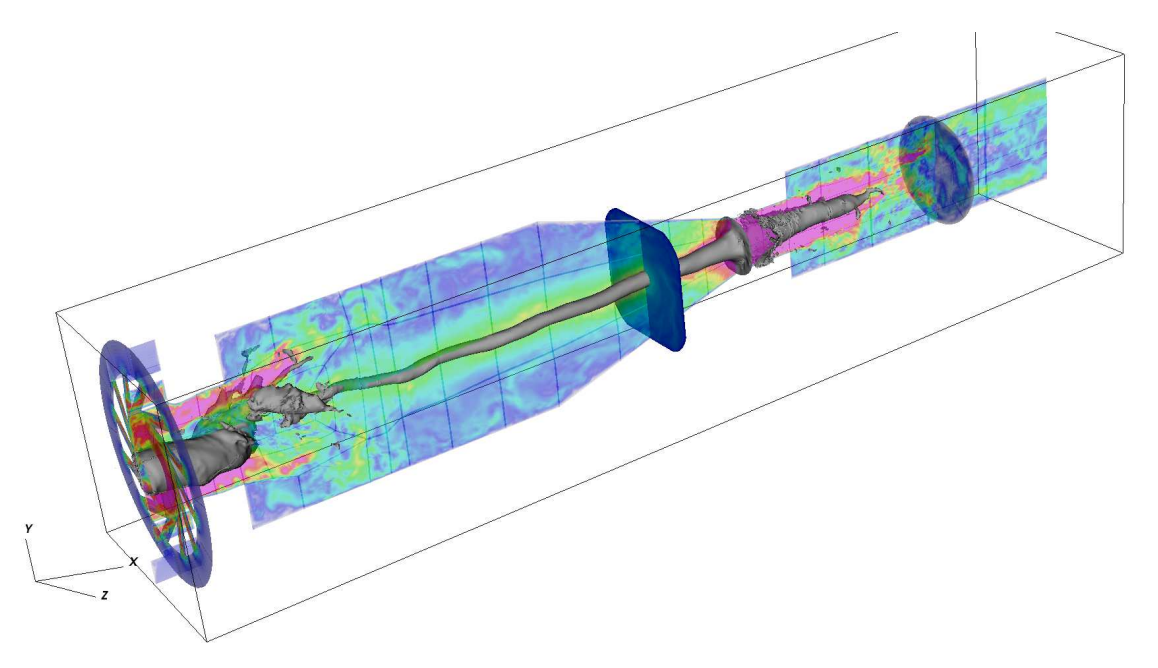

(a) CVC and PVC: iso-pressure contours and velocity magnitude

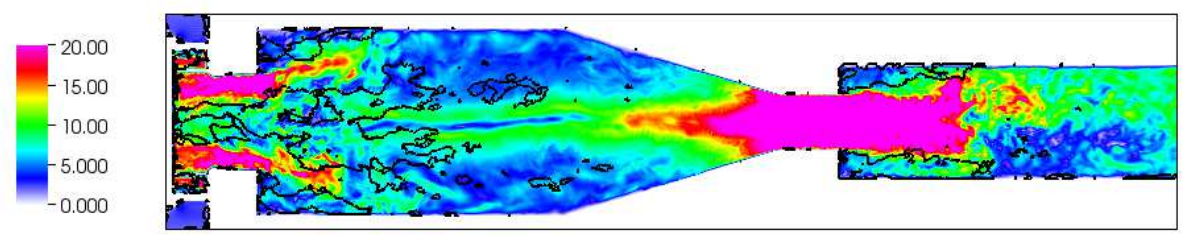

(b) A snapshot of velocity magnitude in the centre plane

Figure 16: The influence of exit domain: Coherent Structures 


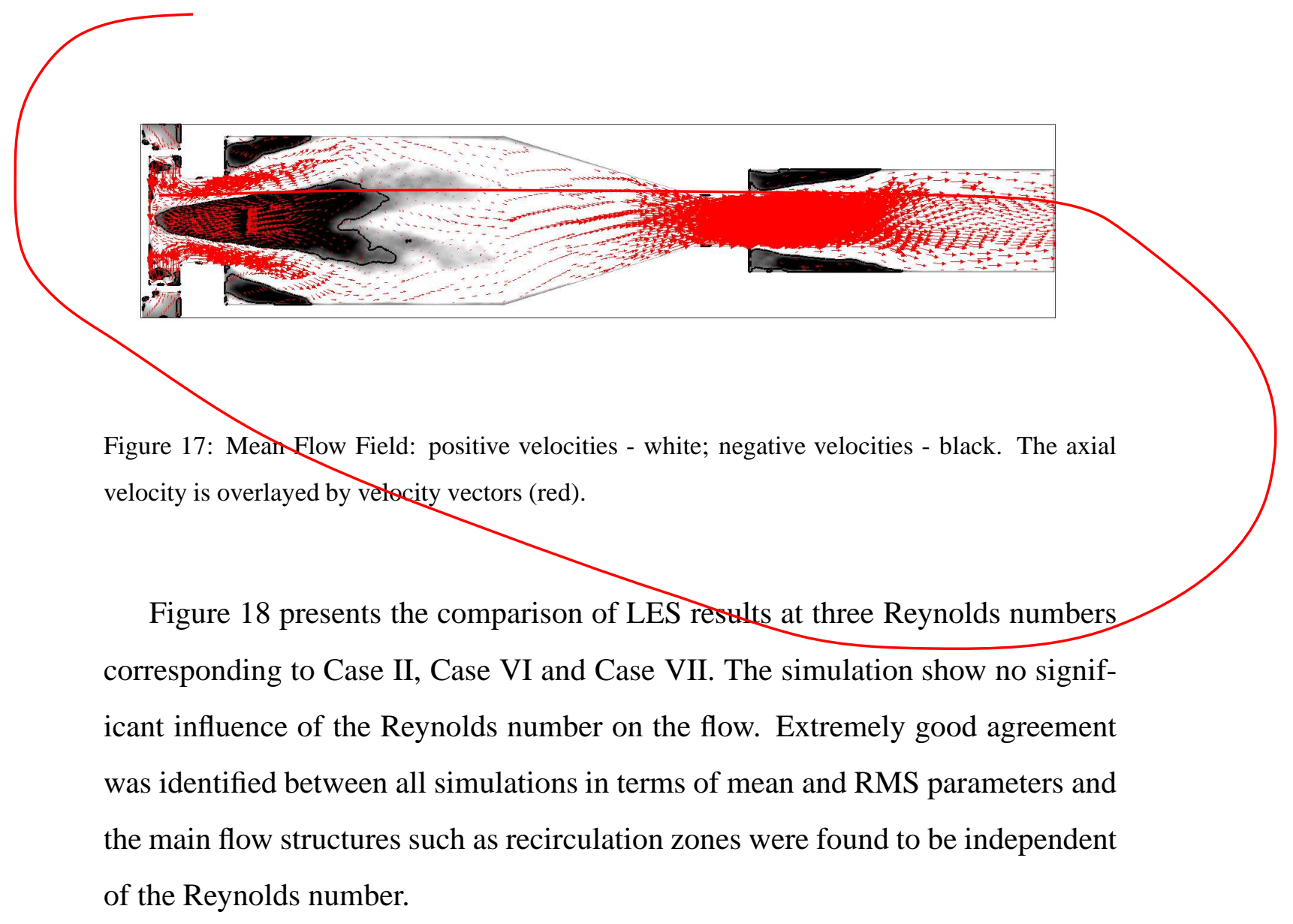

\section{Conclusions}

The LES simulation of the turbulent isothermal flow in a SGT-100 combustor under pressure conditions has been completed. Results from the main reference case were discussed in detail and some attempts to improve the LES results were presented. The influence of the sub- grid turbulence model was discussed as well as the flow dependency on Reynolds number.

The isothermal LES results were found to be in good agreement with experimental data. The flow field was well captured for both the mean and RMS values. 

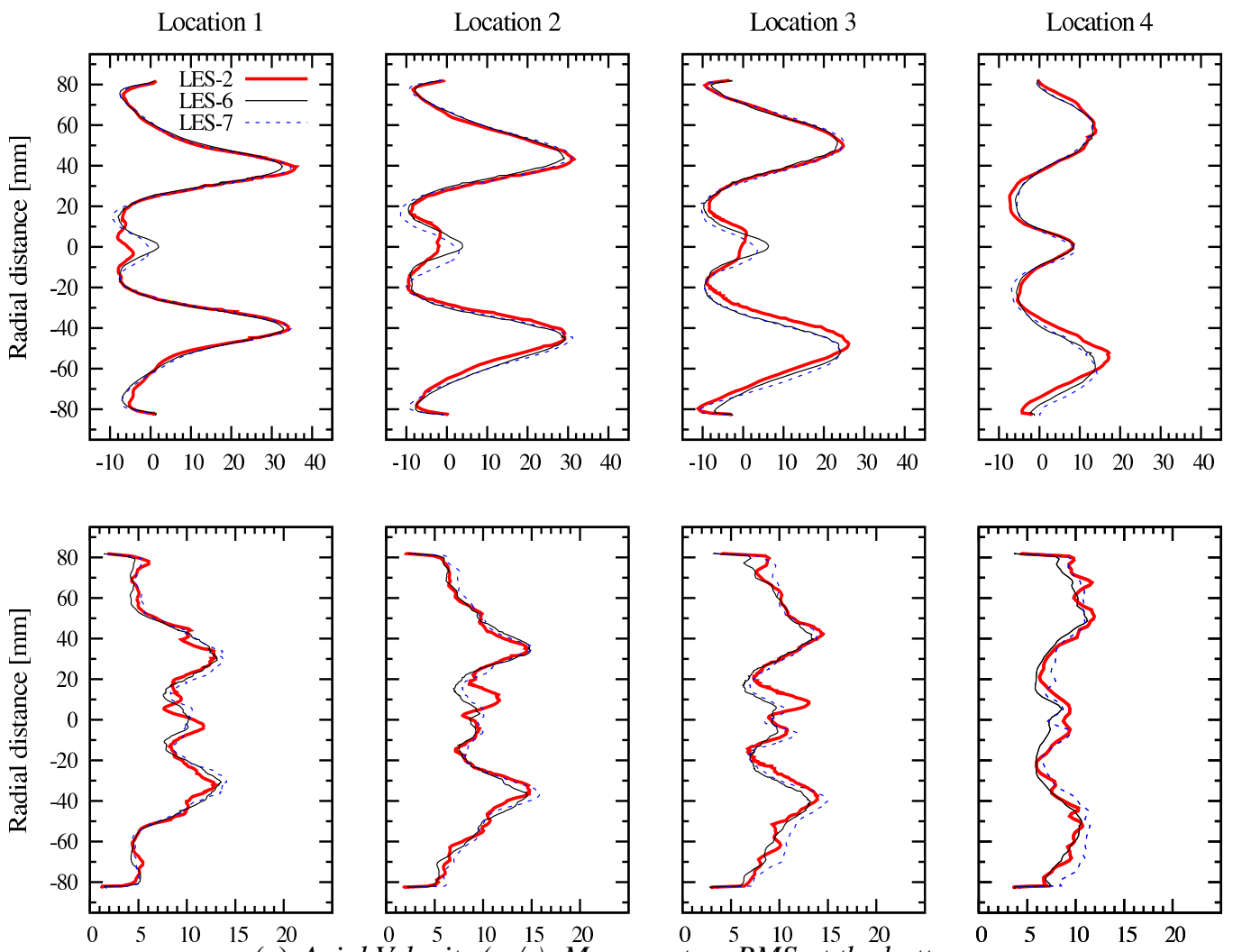

(a) Axial Velocity $(\mathrm{m} / \mathrm{s})$. Mean on top, RMS at the bottom.
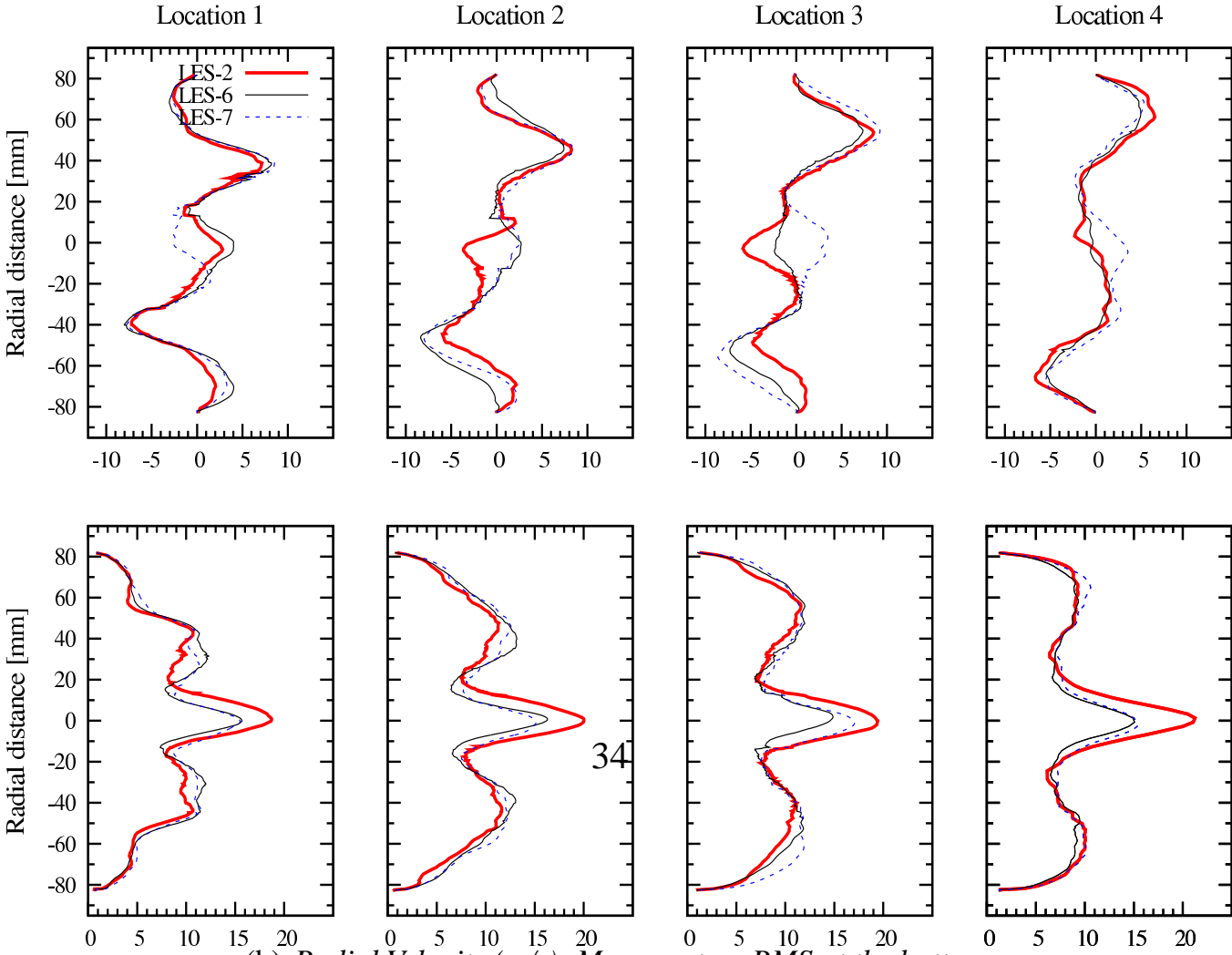

(b) Radial Velocity $(\mathrm{m} / \mathrm{s})$. Mean on top, RMS at the bottom.

Figure 18: The comparison between differing Reynolds number flows. NOTE: LES results from Case II have been scaled to inlet velocity ratio. 
Detailed comparisons with experimental data demonstrate that regions of high vorticity and high shear were correctly located by the LES. Coherent flow structures such as PVC and CVC were observed inside the combustor.

The LES results were found to be effectively independent of the grid. Axial mesh redistribution and axial mesh refinement did not affect the main flow features. Only small improvements were observed in the mean radial velocity component with refinement; however the main flow field and the strength of the exit core was unchanged by axial mesh redistribution or refinement.

The sub-grid turbulence model did not have a significant effect on the flow field. This suggests that majority of the energy containing structures were adequately resolved. For complex wall bounding isothermal flows such as the SGT100 combustor, both tested sub-grid models give similar results and are in good agreement with measured data.

The strength of the CVC was poorly reproduced by the LES. It is suggested that the CVC is associated with exit confinement. An improved outlet boundary condition including part of the exhaust system, was implemented, changing the nature of the outflow condition from sub-critical to supercritical. With the extended domain the CVC extends in the exhaust pipe although its simulated strength remains low. The reasons for this are at present unclear.

\section{Acknowledgement}

This work was supported by SIEMENS Industrial Turbomachinery Ltd. The authors would like to acknowledge the support of Drs U. Stopper, W. Meier, M. Stör, A Bonaldo and V. Sanderson. The support of the UK Turbulence Consortium (UKTC): EPSRC project EP/G069581/1) is also acknowledged for access to 
HECTOR computing facility.

\section{References}

Bulat, G., Jones, W. P., Marquis, A. J., 2013. Large eddy simulation of an industrial gas-turbine combustion chamber using the sub-grid PDF method. Proceedings of the Combustion Institute 34 (2), 3155 - 3164.

Bulat, G., Jones, W. P., Marquis, A. J., 2014. NO and CO formation in an industrial gas-turbine combustion chamber using LES with the eulerian sub-grid PDF method. Combustion and Flame, In press.

Clayton, D., 2006. Large eddy simulation of non-premixed flow in complex geometries. Ph.D. thesis, Imperial College, University of London, UK.

Craft, T., Iacovides, H., Launder, B., 2008. Some Swirling-flow Challenges for Turbulent CFD. Flow, Turbulence and Combustion 80, 419-434.

Dunham, D., Spencer, A., McGuirk, J. J., Dianat, M., 11 2009. Comparison of unsteady reynolds averaged navier-stokes and large eddy simulation computational fluid dynamics methodologies for air swirl fuel injectors. Journal of Engineering for Gas Turbines and Power 131 (1), 011502-011510.

Escudier, M., 1988. Vortex breakdown: observations and explanations. Progress in Aerospace Science 25, 189-229.

Escudier, M. P., Nickson, A. K., Poole, R. J., 2006. Influence of outlet geometry on strongly swirling turbulent flow through a circular tube. Physics of Fluids $18,125103$. 
Franzelli, B., Riber, E., Gicquel, L. Y., Poinsot, T., 2012. Large eddy simulation of combustion instabilities in a lean partially premixed swirled flame. Combustion and Flame 159 (2), $621-637$.

Hall, M. G., 1972. Vortex breakdown. Annual Review of Fluid Mechanics 4, $195-$ 218.

Jochmann, P., Sinigersky, A., Hehle, M., Schäfer, O., Koch, R., Bauer, H.-J., 2006. Numerical simulation of a precessing vortex breakdown. International Journal of Heat and Fluid Flow 27 (2), 192 - 203.

Jones, W. P., di Mare, F., Marquis, A. J., 2002. LES-BOFFIN: Users Guide. Technical Memorandum, Imperial College, London.

Jones, W. P., Lettieri, C., Marquis, A. J., Navarro-Martinez, S., 2012a. Large eddy simulation of the two-phase flow in an experimental swirl-stabilized burner. International Journal of Heat and Fluid Flow 38 (0), 145 - 158.

Jones, W. P., Lyra, S., Navarro-Martinez, S., 2012b. Large eddy simulation of turbulent confined highly swirling annular flows. Flow, Turbulence and Combustion 89 (3), 361-384.

Jones, W. P., Marquis, A. J., Prasad, V. N., 2012c. \{LES $\}$ of a turbulent premixed swirl burner using the eulerian stochastic field method. Combustion and Flame 159 (10), $3079-3095$.

Le, H., Moin, P., Kim, J., 1997. Direct numerical simulation of turbulent flow over a backward facing step. Journal of Fluid Mechanics 330, 349-374. 
Leibovich, S., 1978. The structure of vortex breakdown. Annual Review of Fluid Mechanics 10, 221-246.

Lucca-Negro, O., O’Doherty, T., 2001. Vortex breakdown: a review. Progress in Energy and Combustion Science 27, 431-481.

Meier, W., Weigand, P., Duan, X., Giezendaner-Thoben, R., 2007a. Detailed characterisation of the dynamics of thermoacoustic pulsations in a lean premixed swirl flame. Combustion and Flame 150, 2-26.

Meier, W., Weigand, P., Duan, X., Giezendanner-Thoben, R., 2007b. Detailed characterisation of the dynamics of thermoacoustic pulsations in a lean premixed swirl flame. Combustion and Flame 150 (1-2), 2 - 26.

Moureau, V., Domingo, P., Vervisch, L., 2011. From large-eddy simulation to direct numerical simulation of a lean premixed swirl flame: Filtered laminar flame-pdf modelling. Combustion and Flame 158 (7), 1340 - 1357.

Piomelli, U., Balaras, E., 2002. Wall-layer models for large-eddy simulations. Annual Review of Fluid Mechanics 34 (1), 349-374.

Piomelli, U., Liu, J., Apr. 1995. Large-eddy simulation of rotating channel flows using a localised dynamic-model. Physics of Fluids 7 (4), 839-848.

Poinsot, T., Veynante, D., 2005. Theoretical and Numerical Combustion. Second Edition: R.T. Edwards.

Roux, S., Cazalens, M., Poinsot, T., 2014/04/22 2008. Outlet-boundary-condition influence for large eddy simulation of combustion instabilities in gas turbines. Journal of Propulsion and Power 24 (3), 541-546. 
Roux, S., Lartigue, G., Poinsot, T., Meier, U., Bérat, C., 2005. Studies of mean and unsteady flow in a swirled combustor using experiments, acoustic analysis, and large eddy simulations. Computers \& Fluids 141, 40-54.

Selle, L., Lartigue, G., Poinsot, T., Koch, R., Schildmacher, K.-U., Krebs, W., Prade, B., Kaufmann, P., Veynante, D., 2004. Compressible large eddy simulation of turbulent combustion in complex geometry on unstructured meshes. Computers \& Fluids 137, 489-505.

Squire, H. B., 1962. Analysis of the Vortex breakdown. Akademie Verlag, Berlin, p. 306.

Stopper, U., 2011a. Database of experimental results from TURCHEMI test campaign on SGT-100 at DLR Stuttgart, DLR-Siemens internal database.

Stopper, U., 2011b. TURCHEMI final report, DLR-Siemens internal report.

Stopper, U., 2013. Weiterentwicklung und Anwendung der Laser-RamanStreuung zur Untersuchung industrieller Vormischflammen in einer Hochdruckbrennkammer. Ph.D. thesis, University of Stuttgart.

Stopper, U., Aigner, M., Ax, H., Meier, W., Sadanandan, R., Stöhr, M., Bonaldo, A., 2010. PIV, 2D-LIF, and 1-D Raman measurements of flow field, composition and temperature in premixed gas turbine flames. Experimental Thermal and Fluid Science 34 (3).

Stopper, U., Kim, I. S., Aigner, M., Meier, W., Sadanandan, R., Stöhr, M., 12 2009. Flow field and combustion characterisation of premixed gas turbine flames by planar laser techniques. Journal of Engineering for Gas Turbines and Power 131 (2), 021504-021512. 
Stopper, U., Meier, W., Sadanandan, R., Stöhr, M., Aigner, M., Bulat, G., 2013. Experimental study of industrial gas turbine flames including quantification of pressure influence on flow field, fuel/air premixing and flame shape. Combustion and Flame 160, 2103-2118.

Syred, N., 2006. A review of oscillation mechanisms and the role of the precessing vortex core (PVC) in swirl combustion systems. Progress in Energy and Combustion Science 32, 93-161.

Tennekes, H., Lumley, J. L., 1972. First course in Turbulence. MIT press, Cambridge, Massachussets.

Wang, S., Yang, V., Hsiao, G., Hsieh, S.-Y., Mongia, H. C., 7 2007. Large-eddy simulations of gas-turbine swirl injector flow dynamics. Journal of Fluid Mechanics 583, 99-122.

Weigand, P., Meier, W., Duan, X., Stricker, W., Aigner, M., 2006a. Investigations of swirl flames in a gas turbine model combustor I. Flow field, structures, temperature, and species distributions. Combustion and Flame 144, 205-224.

Weigand, P., Meier, W., Duan, X., Stricker, W., Aigner, M., 2006b. Investigations of swirl flames in a gas turbine model combustor: I. flow field, structures, temperature, and species distributions. Combustion and Flame 144 (1-2), 205 224.

Wolf, P., Staffelbach, G., Roux, A., Gicquel, L., Poinsot, T., Moureau, V., 2009. Massively parallel LES of azimuthal thermo-acoustic instabilities in annular gas turbines. Comptes Rendus Mécanique 337 (6-7), 385 - 394, combustion for aerospace propulsion. 